\title{
Integration of linkage maps for the Amphidiploid Brassica napus and comparative mapping with Arabidopsis and Brassica rapa
}

\author{
Jun Wang ${ }^{1,4}$, Derek J Lydiate ${ }^{2}$, Isobel AP Parkin², Cyril Falentin ${ }^{3}$, Régine Delourme ${ }^{3}$, Pierre WC Carion', \\ Graham J King ${ }^{1,5^{*}}$
}

\begin{abstract}
Background: The large number of genetic linkage maps representing Brassica chromosomes constitute a potential platform for studying crop traits and genome evolution within Brassicaceae. However, the alignment of existing maps remains a major challenge. The integration of these genetic maps will enhance genetic resolution, and provide a means to navigate between sequence-tagged loci, and with contiguous genome sequences as these become available.

Results: We report the first genome-wide integration of Brassica maps based on an automated pipeline which involved collation of genome-wide genotype data for sequence-tagged markers scored on three extensively used amphidiploid Brassica napus $(2 n=38)$ populations. Representative markers were selected from consolidated maps for each population, and skeleton bin maps were generated. The skeleton maps for the three populations were then combined to generate an integrated map for each $L G$, comparing two different approaches, one encapsulated in JoinMap and the other in MergeMap. The BnaWAIT_01_2010a integrated genetic map was generated using JoinMap, and includes 5,162 genetic markers mapped onto 2,196 loci, with a total genetic length of 1,792 cM. The map density of one locus every $0.82 \mathrm{cM}$, corresponding to $515 \mathrm{Kbp}$, increases by at least threefold the locus and marker density within the original maps. Within the B. napus integrated map we identified 103 conserved collinearity blocks relative to Arabidopsis, including five previously unreported blocks. The BnaWAIT_01_2010a map was used to investigate the integrity and conservation of order proposed for genome sequence scaffolds generated from the constituent A genome of Brassica rapa.
\end{abstract}

Conclusions: Our results provide a comprehensive genetic integration of the B. napus genome from a range of sources, which we anticipate will provide valuable information for rapeseed and Canola research.

\section{Background}

Brassica napus is found almost solely in an agricultural setting represented by the oil crops oilseed rape (Canola, rapeseed) and vegetable/fodder crops swede and rutabaga. As one of the most commercially important oil crops, it is grown in most temperate regions of the world including North and South America, Europe, Australia, and East and South Asia, for the production of vegetable oil for human consumption, industrial uses

\footnotetext{
* Correspondence: graham.king@bbsrc.ac.uk

'Department of Plant Sciences, Rothamsted Research, Harpenden, AL5 2JQ, UK Full list of author information is available at the end of the article
}

including as a lubricant or biofuel, and a protein meal used as animal feed.

Brassica napus is an amphidiploid species (AC genome, $\mathrm{n}=19$ ) derived from a recent hybridization event between Brassica rapa (A genome, $\mathrm{n}=10$ ) and Brassica oleracea (C genome, $n=9)(U, 1935)$. It probably arose and was selected in human cultivation within the past 10,000 years. It is widely accepted that Brassica species diverged from a common ancestor with the Arabidopsis lineage 20 MYA [1,2]. Similarly, the A and C genomes diverged from a common ancestor $\sim 5$ MYA. Since the divergence of the two lineages leading to the genera Brassica and Arabidopsis, there has been a triplication event that created a hexaploid ancestor unique to the
C Biomed Central 
tribe Brassiceae [3-7]. This is supported by evidence from $\sim 1,300$ restriction fragment length polymorphism (RFLP) loci in the Brassica A and C genomes that were mapped to homologous positions in Arabidopsis [7], along with evidence from comparative linkage mapping between B. juncea, B. oleracea, B. rapa and Arabidopsis [8-11] and FISH analysis [6]. These events occurred after ancient whole-genome duplications found in Arabidopsis ancestors (1-3R, or $\gamma, \beta$ and $\alpha$, respectively) [12-14]. A recent study of the distribution and rate of synonymous substitutions in homologous sequences among Brassica and Arabidopsis has suggested that the triplicated B. rapa (A) genome may also have undergone a process of genome shrinkage [15].

Genetic linkage maps represent a key resource to understand genome organisation, evolutionary relationships, and to assist in the assignment and orientation of sequence assemblies to correct chromosome locations. In addition, dense linkage maps provide the basis for map-based cloning of major genes and QTLs underlying agronomic traits, as well as for marker-assisted selection. In $B$. napus, a range of sequence tagged genetic markers, including restriction fragment length polymorphism (RFLPs), simple sequence repeats (SSRs) and single nucleotide polymorphisms (SNPs) have been developed both from Arabidopsis and Brassica species. Various versions of linkage maps, derived from a range of reference $B$. napus mapping populations, have been published within the last twenty years [7,16-26].

Development of a high density integrated genetic map of B. napus derived from well established mapping populations will provide a superior tool for high resolution mapping and verification of DNA sequence contig order and orientation. Benefits arise from incorporating information derived from the increased number of individuals and chiasmata represented within the populations. Since the parent lines are genetically diverse, a larger proportion of markers will be informative and so enable a higher number of mapped markers to be obtained from the potential number of markers available. For several crop species such as maize $[27,28]$, soybean $[29,30]$, barley [31-33], sorghum [34-36], wild wheat $[37,38]$, grapevine [39,40], cowpea [41] and peanut [42], integrated consensus linkage maps of multiple mapping populations have been developed. In Brassica, early attempts [43] to align linkage maps derived from different Brassica populations were based on very low numbers of shared markers, and suffered from lack of resolution with respect to distinguishing between paralogous loci. More recent efforts have been successful in generating aligned maps for the Brassica A genome that integrate marker information using a common set of SSRs scored in B. rapa and B. napus [26].
Although conceptually simple, in practice construction of an integrated map from diverse sources (populations and types of markers) is a non-trivial exercise. This is particularly true where genetic maps have been generated from different populations or sub-populations with different subsets of informative genetic markers. The situation is exacerbated where multiple paralogous loci may exist as a result of chromosomal segmental duplication over relatively recent evolutionary time, which in the case of B. napus is compounded by amphidiploidy. This may lead to a low number of shared (bridge or anchor) markers between maps. Moreover, the quality of genotype data may vary across studies, thus hampering the progress of genetic map integration.

Several systematic approaches have been proposed to construct integrated maps. Early attempts involved pooling genotype information from several segregating populations, and then relying on conventional mapping algorithms (e.g., log-likelihood statistic) to build a single composite map $[44,45]$. However, this method has some shortcomings. Firstly, mapping populations may be of different types (e.g., double haploid, backcross, F2 intercross and recombinant inbred lines) and have different estimates of genetic distance. Pooling information cannot be applied to all combinations of populations, since treating data from different sources equivalently is flawed. Secondly, once a composite genotype matrix is generated from several populations it contains a large proportion of missing data, where conventional mapping algorithms will tend to generate maps of low quality. Alternative approaches have involved modification to mapping algorithms, such as employed by JoinMap [46-48] and Carthagène [49]. These software packages take into account all available information from each individual dataset (e.g., population structure and size) and estimate the marker order and genetic distances of common (anchor or bridge) markers using regression mapping (JoinMap) or multiple 2-point maximum likelihood (Carthagène). Since both methods involve exhaustive search of objective functions, the computational process to search for an optimal map is very time consuming. This becomes limiting for map integration that involves a very large number of markers and/or populations. A third approach, MergeMap [50], relies on graph theory $[51,52]$ and uses directed acyclic graphs (DAGs) to represent maps from individual populations, and to resolve conflicts between maps. Although MergeMap does not make use of genotype data, simulations have shown that MergeMap can outperform JoinMap in terms both of accuracy and running time [50].

In this study, we report the first genome-wide integration of Brassica genetic maps based on an automated implementation of a defined algorithm. We selected three extensively studied $B$. napus DH mapping 
populations, BnaSNDH, BnaSGDH and BnaDYDH, since they share a high number of loci derived from common genetic marker assays. A range of different published and unpublished sources of genotype data have been collated and curated for each population. Our approach involved first constructing a population-specific consolidated map by merging constituent genotype matrices for each mapping population following initial assignment to each of the 19 LGs. A skeleton map that consists solely of representative markers from each bin was then prepared for the subsequent map integration for each population. We were able to compare the contrasting approaches employed by JoinMap and MergeMap, and then to investigate models of genome collinearity within the Brassicaceae, and the relationship between genetic and physical distances.

\section{Results}

The first stage of the integration process involved combining map data from previously published sources with new genotype score datasets, primarily from a large number of SSR markers for each of the three DH populations. This not only increases the map density and represents more recombination events, but also for the purpose of map integration potentially provides additional 'bridge' information between populations.

\section{Population-specific consolidated maps for three DH populations}

BnaSGDH_03_2010a is the first published map derived from the BnaSGDH population, and includes 483 RFLP and 1,897 SSR marker loci. In addition to 1,287 RFLP markers used previously in the BnaSNDH population $[7,16], 1,314$ SSR markers were included in the BnaSNDH_05_2010a consolidated map. In the BnaDYDH_05_2010a map, there were 356 SSR and 511 other genetic markers, including RFLPs, AFLPs, RAPDs and SNPs. The population specific genetic maps comprised 745 (BnaSNDH), 894 (BnaSGDH) and 528 (BnaDYDH) unique mapping loci (Table 1). The elimination of unlikely local double crossovers and selection of representative markers to form population-specific bin maps greatly reduced the initial inflated lengths of the LGs, by up to $50 \%$, with average LG lengths varying from 140 to $194 \mathrm{cM}$ in the three mapping populations. The lengths of LGs among all three population-specific maps were positively correlated (between BnaSNDH and BnaSGDH Spearman's correlation $r=0.68, p=0.0016$; between BnaSNDH and BnaDYDH $r=0.55, p=0.02$; and between BnaSGDH and BnaDYDH $r=0.49, p=0.03$,).

\section{Segregation distortion within the three DH populations} Comparison of the three DH populations indicated that the proportion of mapped loci displaying segregation distortion ( $p<0.05$ in the $\chi^{2}$ test) varied from $22 \%$ to $49 \%$ (Table 2). The proportion of loci showing segregation distortion within the BnaSNDH_03_2005a map [7], $18.3 \%$, was slightly lower than that within our consolidated BnaSNDH map BnaSNDH_05_2010a.

The most extreme segregation distortion in BnaSNDH was observed in LG A03, with 31 out of 62 loci (50\%) mostly clustered in the top arm. The BnaSNDH A03 showed an average skewed ratio of 1.65:1 over its entire length $\left(\chi^{2}=174.02, p<0.0001\right)$, favouring alleles from SYN1, the female parent. In BnaSGDH, several LGs showed segregation distortion along almost the entire lengths (> $80 \%$ of the LG length). For example, all 32 loci in C06 showed segregation distortion (a skewed ratio of 4.39:1 over the entire length, $\chi^{2}=758.41, p<$ 0.0001 ), favouring alleles from female line PSA12. In BnaDYDH, the most extreme case of segregation distortion was found on A02 where 21 out of 22 loci (95.4\%) showed segregation distortion, favouring alleles from the male parental line Yudal. The BnaDYDH A02 showed an average skewed ratio of 1:1.85 instead of 1:1 over its entire length $\left(\chi^{2}=161.04, \mathrm{p}<0.0001\right)$.

\section{Conservation of marker orders between populations}

Comparison of marker orders between the three population-specific consolidated maps indicated good agreement over most of the LGs (Additional File 1 generated by MapChart 2.1). Marker orders were strongly positively correlated between BnaSNDH and BnaSGDH, with a mean correlation coefficient of 0.88 (Table 3). A07 was an exception $(p=0.09)$. This could result from an observed inversion between BnaSGDH and the other two maps on A07 (Additional File 1). For some LGs, the number of shared markers was very low between populations (e.g., $\leq 3$ shared markers for 7 LGs between BnaSNDH and BnaDYDH, and for 4 LGs between BnaSGDH and BnaDYDH, Table 1). In these cases it was difficult to judge the overall consistency of marker order among maps, as reported by correlation coefficients. Thus there was no significant correlation reported with many BnaDYDH LGs. However, marker order was conserved within those LGs where sufficient bridge markers (more than 4 shared markers) allowed for assessment of statistical significance (Table 3). This provided more confidence for the subsequent use of bridge markers for the map integration.

\section{Integration of genetic maps using JoinMap and comparison with population-specific maps}

The BnaWAIT_01_2010a integrated linkage map contains 5,162 markers representing 2,196 unique loci (i.e. unique map positions and bins) (Table 1, Additional File 2 and 3). Map integration using JoinMap 4.0 was based on representative markers from the population-specific 
Table 1 Distribution of marker loci (n), shared markers (n), unique mapping loci (n) and LGs lengths (cM) within different LGs of the three population-specific B. napus maps, BnaSNDH_05_2010a (Map A), BnaSGDH_03_2010a (Map B) and BnaDYDH_05_2010a (Map C) and the two integrated maps, BnaWAIT_01_2010a (Map D) generated by JoinMap and BnaWAIT_01_2010b (Map E) generated by MergeMap

\begin{tabular}{|c|c|c|c|c|c|c|c|c|c|c|c|c|c|c|c|c|c|}
\hline \multirow[b]{2}{*}{ LG } & \multicolumn{4}{|c|}{ Number of markers (n) } & \multicolumn{3}{|c|}{ Number of shared markers (n) } & \multicolumn{5}{|c|}{ Number of unique loci (n) } & \multicolumn{5}{|c|}{ LGs length (cM) } \\
\hline & $\begin{array}{r}\text { Map } \\
\text { A }\end{array}$ & $\begin{array}{r}\text { Map } \\
\text { B }\end{array}$ & $\begin{array}{r}\text { Map } \\
C\end{array}$ & $\begin{array}{r}\text { Map } \\
\text { D \& E }\end{array}$ & $\begin{array}{r}\text { Map } \\
\text { A/B }\end{array}$ & $\begin{array}{r}\text { Map } \\
\mathrm{A} / \mathrm{C}\end{array}$ & $\begin{array}{c}\text { Map } \\
B / C\end{array}$ & $\begin{array}{r}\text { Map } \\
\text { A }\end{array}$ & $\begin{array}{r}\text { Map } \\
\text { B }\end{array}$ & $\begin{array}{r}\text { Map } \\
\text { C }\end{array}$ & $\begin{array}{r}\text { Map } \\
\text { D }\end{array}$ & $\begin{array}{r}\text { Map } \\
\mathbf{E}\end{array}$ & $\begin{array}{r}\text { Map } \\
\text { A }\end{array}$ & $\begin{array}{r}\text { Map } \\
B\end{array}$ & $\begin{array}{r}\text { Map } \\
\text { C }\end{array}$ & $\begin{array}{r}\text { Map } \\
\text { D }\end{array}$ & $\begin{array}{r}\text { Map } \\
E\end{array}$ \\
\hline A01 & 145 & 107 & 60 & 271 & 26 & 9 & 8 & 54 & 52 & 33 & 134 & 98 & 265.31 & 227.47 & 159.66 & 100.96 & 440.76 \\
\hline A02 & 131 & 70 & 34 & 199 & 30 & 6 & 2 & 45 & 25 & 22 & 92 & 72 & 154.07 & 129.59 & 121.95 & 86.69 & 194.45 \\
\hline A03 & 199 & 166 & 72 & 385 & 39 & 9 & 7 & 62 & 63 & 44 & 174 & 141 & 222.34 & 247.10 & 217.88 & 126.10 & 417.02 \\
\hline A04 & 83 & 83 & 33 & 176 & 18 & 1 & 4 & 26 & 28 & 20 & 77 & 64 & 108.13 & 157.47 & 111.51 & 80.32 & 216.89 \\
\hline A05 & 113 & 76 & 41 & 205 & 19 & 4 & 3 & 31 & 40 & 25 & 87 & 74 & 123.98 & 206.19 & 123.15 & 100.15 & 213.32 \\
\hline A06 & 145 & 114 & 43 & 261 & 33 & 4 & 4 & 33 & 52 & 30 & 111 & 93 & 158.92 & 149.34 & 158.26 & 108.46 & 201.08 \\
\hline $\mathrm{A} 07$ & 95 & 100 & 33 & 211 & 11 & 3 & 4 & 42 & 43 & 24 & 102 & 91 & 220.08 & 140.65 & 92.85 & 88.08 & 269.32 \\
\hline A08 & 106 & 78 & 41 & 199 & 17 & 5 & 4 & 28 & 20 & 26 & 80 & 64 & 130.32 & 87.97 & 102.84 & 73.77 & 147.75 \\
\hline A09 & 148 & 154 & 70 & 327 & 29 & 8 & 8 & 52 & 68 & 36 & 153 & 131 & 236.60 & 256.02 & 154.03 & 103.21 & 454.28 \\
\hline A10 & 134 & 74 & 40 & 215 & 23 & 7 & 5 & 22 & 37 & 26 & 89 & 73 & 127.17 & 111.02 & 147.12 & 78.69 & 219.01 \\
\hline C01 & 117 & 141 & 50 & 280 & 19 & 4 & 8 & 32 & 70 & 23 & 126 & 104 & 220.52 & 263.74 & 126.82 & 94.59 & 308.63 \\
\hline $\mathrm{CO} 2$ & 121 & 88 & 44 & 232 & 14 & 5 & 3 & 27 & 37 & 29 & 90 & 83 & 113.53 & 106.79 & 116.85 & 67.09 & 255.17 \\
\hline $\mathrm{CO3}$ & 255 & 253 & 64 & 497 & 57 & 6 & 13 & 68 & 75 & 45 & 200 & 169 & 266.69 & 317.20 & 240.38 & 132.94 & 516.25 \\
\hline $\mathrm{CO} 4$ & 173 & 189 & 61 & 379 & 36 & 2 & 7 & 33 & 51 & 40 & 137 & 107 & 163.42 & 197.08 & 161.66 & 111.41 & 307.20 \\
\hline $\mathrm{CO5}$ & 142 & 141 & 42 & 284 & 30 & 5 & 7 & 41 & 39 & 21 & 110 & 79 & 160.35 & 203.83 & 164.19 & 88.80 & 237.87 \\
\hline C06 & 34 & 83 & 30 & 133 & 10 & 2 & 2 & 16 & 32 & 17 & 67 & 65 & 105.63 & 90.39 & 89.98 & 62.07 & 204.02 \\
\hline $\mathrm{CO7}$ & 150 & 153 & 42 & 303 & 30 & 3 & 12 & 38 & 45 & 28 & 117 & 86 & 180.04 & 225.58 & 139.25 & 90.83 & 278.70 \\
\hline C08 & 145 & 141 & 39 & 290 & 28 & 2 & 5 & 46 & 54 & 20 & 117 & 90 & 141.09 & 303.54 & 141.27 & 92.96 & 346.12 \\
\hline CO9 & 165 & 169 & 28 & 315 & 41 & 2 & 4 & 49 & 63 & 19 & 133 & 112 & 193.31 & 258.75 & 97.31 & 104.75 & 319.60 \\
\hline total & 2601 & 2380 & 867 & 5162 & 510 & 87 & 110 & 745 & 894 & 528 & 2196 & 1796 & 3291.51 & 3679.72 & 2666.96 & 1791.87 & 5547.44 \\
\hline
\end{tabular}

bin-maps, including 20\% of all markers as bridge markers across populations (Additional File 3). The A genome is represented by 2,449 markers and the $C$ genome 2,713 (Table 1). The total genetic length for the integrated maps is 1,792 cM, with a mean length of 94.3 cM per LG. The lengths of LGs for BnaWAIT_01_2010a in relation to all three population-specific maps were significantly positively correlated (for BnaSNDH Spearman's correlation $r=0.74, p=0.0005$; for BnaSGDH $r=0.74, p=0.0004$; for BnaDYDH $r=0.68, p=0.002$ ). Although on average there are 2.3 markers per map interval, this ranges from one to 20 . The mean map density is a locus per $0.82 \mathrm{cM}(1,792 \mathrm{cM} / 2,196$ positions). This corresponds to a locus every $515 \mathrm{Kbp}$, based on the estimated size of $1,132 \mathrm{Mbp}[53,54]$ for the $B$. napus genome. The distribution of map intervals was highly skewed, with a preponderance of shorter distances (Figure 1). The marker density was 1 marker every $0.35 \mathrm{cM}(1,792 \mathrm{cM} / 5,162$ markers $)$, or 1 marker every $219 \mathrm{Kbp}$ (1,132 Mbp/5,162 markers).

Comparison of the marker order between the population-specific and integrated maps indicated overall good agreement (Figure 2; Additional File 4; Table 3). For 11 LGs, there was good agreement between the integrated and population-specific maps (Spearman's correlation $r>0.90$ for all three pairwise comparisons). For a further five LGs the agreement in marker order was good for two of the pairwise comparisons $(r>0.90)$. For A07, A08, C05 and C06 there was a relatively low level of agreement, although the marker order was still significantly positively correlated between the three component and integrated maps. This could be due to the local order discrepancies between component maps. When there are inversions in specific populations, the use of an integrated map alone may not be informative. Map alignment of different populations (presented in Additional File 1) and dot-plots (presented in Figure 2 and Additional File 4) became powerful tools to indicate genetic regions where maker order differs among population-specific maps.

Since there were very few markers in common between some LGs, it implied that the integrated map BnaWAIT_01_2010a was likely the best estimate of a map. JoinMap 4.0 generates two alternative maps (Round 1 and Round 2 under the algorithm of regression) where a group of poorly fitting representative markers in the skeleton map were excluded from the analyses. We reported the two alternative integrated maps and the Spearman's rank correlation test between these two integrated maps and population-specific maps for all 19 LGs (Additional File 5). 
Table 2 Segregation distortion within the three $B$. napus DH populations, BnaSNDH, BnaSGDH and BnaDYDH

\begin{tabular}{|c|c|c|c|c|c|c|}
\hline \multirow{3}{*}{ LG } & \multicolumn{2}{|c|}{ BnaSNDH } & \multicolumn{2}{|c|}{ BnaSGDH } & \multicolumn{2}{|c|}{ BnaDYDH } \\
\hline & \multicolumn{2}{|c|}{$\begin{array}{l}\text { Number of loci } \\
\text { showing } \\
\text { segregation } \\
\text { distortion } \\
\text { favouring }\end{array}$} & \multicolumn{2}{|c|}{$\begin{array}{l}\text { Number of loci } \\
\text { showing } \\
\text { segregation } \\
\text { distortion } \\
\text { favouring }\end{array}$} & \multicolumn{2}{|c|}{$\begin{array}{l}\text { Number of loci } \\
\text { showing } \\
\text { segregation } \\
\text { distortion } \\
\text { favouring }\end{array}$} \\
\hline & female & male & female & Male & female & Male \\
\hline A01 & 5 & 5 & 0 & 5 & 8 & 2 \\
\hline $\mathrm{A} 02$ & 4 & 6 & 0 & 17 & 0 & 21 \\
\hline A03 & 31 & 0 & 12 & 0 & 23 & 0 \\
\hline A04 & 2 & 0 & 12 & 2 & 1 & 1 \\
\hline A05 & 1 & 0 & 1 & 6 & 0 & 13 \\
\hline A06 & 0 & 0 & 1 & 3 & 0 & 26 \\
\hline A07 & 1 & 1 & 0 & 36 & 5 & 6 \\
\hline A08 & 6 & 0 & 0 & 2 & 2 & 0 \\
\hline A09 & 0 & 6 & 64 & 0 & 19 & 6 \\
\hline A10 & 0 & 7 & 24 & 1 & 2 & 1 \\
\hline C01 & 13 & 0 & 13 & 5 & 7 & 0 \\
\hline $\mathrm{CO2}$ & 3 & 0 & 35 & 0 & 19 & 0 \\
\hline $\mathrm{CO3}$ & 11 & 1 & 0 & 31 & 1 & 36 \\
\hline C04 & 10 & 0 & 1 & 31 & 10 & 0 \\
\hline C05 & 12 & 4 & 35 & 0 & 12 & 0 \\
\hline $\mathrm{CO6}$ & 11 & 0 & 32 & 0 & 15 & 0 \\
\hline $\mathrm{CO7}$ & 0 & 8 & 0 & 8 & 4 & 4 \\
\hline $\mathrm{CO8}$ & 2 & 8 & 28 & 0 & 0 & 4 \\
\hline Co9 & 9 & 0 & 17 & 31 & 0 & 16 \\
\hline total & 121 & 46 & 275 & 178 & 132 & 136 \\
\hline
\end{tabular}

Loci that showed segregation distortion were detected by the calculation of locus genotype frequency where the $\chi^{2}$ test is significant $(p<0.05)$

The BnaWAIT_01_2010a integrated map appeared to be the best estimate of the integrated map for almost all 19 LGs, compared with the other two alternative maps, except for LG C05. But 17 and 10 poorly linked representative markers were excluded from the two alternative integrated maps for C05, respectively (Additional File 5). In general, the marker orders were much conserved $(r>$ 0.95) among all three integrated maps generated by JoinMap (all three rounds).

\section{Integration of genetic maps using MergeMap and comparison with JoinMap}

We compared the pipeline incorporating JoinMap with that using MergeMap. The integrated map produced by MergeMap, BnaWAIT_01_2010b, had a total genetic length of 5,547 cM, consisting of 1,796 loci (Table 1). The map density was thus one map position every 630 Kbp, lower than that produced by JoinMap (one position every $515 \mathrm{Kbp}$ ). Compared with JoinMap, MergeMap tended to generate integrated maps with much higher consistency of marker order compared with each population-specific map, with Spearman's correlation coefficients $>0.95$ across all LGs for all three populations (Table 3).

Comparison and calculation of the Spearman's rank correlation in the marker orders for the integrated maps generated by JoinMap and MergeMap (Table 3) indicated a good agreement between the two methods for most of the LGs. Fifteen LGs had Spearman's correlation coefficients $>0.90$. Not surprisingly, the four LGs with correlation coefficients $<0.90$ were those where JoinMap performed relatively poorly for the map integration (A07, A08, C05 and C06). MergeMap appeared to outperform JoinMap in terms of marker order consistency between integrated maps and population-specific maps (especially for A07, A08, C05 and C06). One should note that MergeMap achieved this by relying solely on the existing marker orders for each component maps, rather than making use of the information within the genotype data to perform the map re-calculation. It is clear that JoinMap tended to produce more accurate estimates of genetic distances and resolve a greater number of unique marker loci for each LG compared with MergeMap (Table 1).

\section{Comparative mapping of B. napus and Arabidopsis, and resolution of collinearity blocks}

Since the BnaWAIT_01_2010a integrated map increased the marker density by more than 3 fold compared with the BnaSNDH_03_2005a map [7], we were able to refine the recognised collinearity blocks and resolve additional blocks within the $B$. napus genome. Sequence data were obtained for RFLPs and 'BBSRC', 'Celera' and 'AAFC' SSR canonical marker assays. Homologous loci were identified within the Arabidopsis genome (Additional File 3 and 6).

We incorporated previously calculated homology results for 99 RFLP markers (prefixed 'es', 'I', 'N', 'R', 'T' and ' $Z$ ') from Parkin et al. [7], which had been established with slightly less stringent criteria. We also identified homologous loci within sequenced $B$. rapa $\mathrm{BAC}$ clones for RFLP and SSR canonical markers, and used the annotation of $984 \mathrm{~B}$. rapa BAC clones (Brassica Genome Gateway: http://brassica.bbsrc.ac.uk/) to infer the putative Arabidopsis gene homology for markers whose relationship to Arabidopsis sequence could not be identified directly. However, this only increased the proportion of markers in the integrated map with homology in Arabidopsis by $1.0 \%$. Local marker order was rearranged for $2.8 \%$ of markers based on physical proximity within sequenced $B$. rapa BAC clones. Additional homology information was obtained for some PCR markers designed from Arabidopsis sequences mapped in BnaDYDH (ACGM from Fourmann et al. [55] and specific PCR markers prefixed 'At', Delourme et al. [25]). In total, $41.0 \%$ of all genetic markers in the 
Table 3 Spearman's rank correlation $(r)$ of the marker order for the comparison among the three population-specific B. napus maps, BnaSNDH_05_2010a, BnaSGDH_03_2010a and BnaDYDH_05_2010a, comparison between each of the three population-specific maps and each of the two integrated maps, BnaWAIT_01_2010a generated by JoinMap and BnaWAIT_01_2010b generated by MergeMap and comparison between the two integrated maps, BnaWAIT_01_2010a and BnaWAIT_01_2010b

\begin{tabular}{|c|c|c|c|c|c|}
\hline LG & Map & BnaSNDH_05_2010a & BnaSGDH_03_2010a & BnaDYDH_05_2010a & BnaWAIT_01_2010a \\
\hline \multirow[t]{4}{*}{$\mathrm{A} 01$} & BnaSGDH_03_2010a & $0.96^{* * * *}$ & & & \\
\hline & BnaDYDH_05_2010a & $0.97^{* * *}$ & $0.97^{* * *}$ & & \\
\hline & BnaWAIT_01_2010a & $0.97^{* * * *}$ & $0.98^{* * * *}$ & $0.99 * * * *$ & \\
\hline & BnaWAIT_01_2010b & $0.99 * * * *$ & $1.00^{* * * *}$ & $1.00^{* * * *}$ & $0.97^{* * *}$ \\
\hline \multirow[t]{4}{*}{ A02 } & BnaSGDH_03_2010a & $0.95^{* * * *}$ & & & \\
\hline & BnaDYDH_05_2010a & $0.94^{*}$ & 1.00 & & \\
\hline & BnaWAIT_01_2010a & $0.99^{* * * *}$ & $0.99^{* * * *}$ & $1.00^{* * * *}$ & \\
\hline & BnaWAIT_01_2010b & $1.00^{* * * *}$ & $0.99^{* * * *}$ & $1.00^{* * * *}$ & $0.99 * * * *$ \\
\hline \multirow[t]{4}{*}{$\mathrm{A} 03$} & BnaSGDH_03_2010a & $0.99 * * * *$ & & & \\
\hline & BnaDYDH_05_2010a & $0.97^{* * *}$ & $0.96^{* *}$ & & \\
\hline & BnaWAIT_01_2010a & $1.00^{* * * *}$ & $0.99 * * * *$ & $1.00 * * * *$ & \\
\hline & BnaWAIT_01_2010b & $1.00^{* * * *}$ & $1.00^{* * * *}$ & $0.98^{* * * *}$ & $0.99 * * * *$ \\
\hline \multirow[t]{4}{*}{ A04 } & BnaSGDH_03_2010a & $0.93^{* * * *}$ & & & \\
\hline & BnaDYDH_05_2010a & -——- & 0.20 & & \\
\hline & BnaWAIT_01_2010a & $0.99 * * * *$ & $0.97^{* * * *}$ & $0.86^{* * * *}$ & \\
\hline & BnaWAIT_01_2010b & $1.00^{* * * *}$ & $1.00^{* * * *}$ & $0.98^{* * * *}$ & $0.95 * * * *$ \\
\hline \multirow[t]{4}{*}{ A05 } & BnaSGDH_03_2010a & $0.90^{* * * *}$ & & & \\
\hline & BnaDYDH_05_2010a & 0.20 & 1.00 & & \\
\hline & BnaWAIT_01_2010a & $0.94^{* * * *}$ & $0.97^{* * * *}$ & $1.00^{* * * *}$ & \\
\hline & BnaWAIT_01_2010b & $1.00^{* * * *}$ & $0.99^{* * * *}$ & $1.00^{* * * *}$ & $0.96^{* * * *}$ \\
\hline \multirow[t]{4}{*}{ A06 } & BnaSGDH_03_2010a & $0.84^{* * * *}$ & & & \\
\hline & BnaDYDH_05_2010a & 1.00 & 1.00 & & \\
\hline & BnaWAIT_01_2010a & $0.96^{* * * *}$ & $0.94^{* * * *}$ & $1.00^{* * * *}$ & \\
\hline & BnaWAIT_01_2010b & $0.97^{* * * *}$ & $0.98^{* * * *}$ & $1.00^{* * * *}$ & $0.95^{* * * *}$ \\
\hline \multirow[t]{4}{*}{ A07 } & BnaSGDH_03_2010a & 0.54 & & & \\
\hline & BnaDYDH_05_2010a & 1.00 & -0.80 & & \\
\hline & BnaWAIT_01_2010a & $0.87^{* * * *}$ & $0.76^{* * * *}$ & $1.00^{* * * *}$ & \\
\hline & BnaWAIT_01_2010b & $1.00^{* * * *}$ & $0.98^{* * * *}$ & $0.98^{* * * *}$ & $0.85^{* * * *}$ \\
\hline \multirow[t]{4}{*}{$\mathrm{A08}$} & BnaSGDH_03_2010a & $0.98^{* * * *}$ & & & \\
\hline & BnaDYDH_05_2010a & 0.70 & 0.80 & & \\
\hline & BnaWAIT_01_2010a & $0.79 * * * *$ & $0.46^{* * * *}$ & $0.58^{* * * *}$ & \\
\hline & BnaWAIT_01_2010b & $1.00^{* * * *}$ & $0.98^{* * * *}$ & $0.98^{* * * *}$ & $0.64^{* * * *}$ \\
\hline \multirow[t]{4}{*}{ A09 } & BnaSGDH_03_2010a & $0.75^{* * * *}$ & & & \\
\hline & BnaDYDH_05_2010a & $0.95^{* * *}$ & $0.93^{* *}$ & & \\
\hline & BnaWAIT_01_2010a & $0.86^{* * * *}$ & $0.91^{* * * *}$ & $0.97^{* * * *}$ & \\
\hline & BnaWAIT_01_2010b & $0.96^{* * * *}$ & $0.99^{* * * *}$ & $1.00^{* * * *}$ & $0.92^{* * * *}$ \\
\hline \multirow[t]{4}{*}{ A10 } & BnaSGDH_03_2010a & $0.97^{* * * *}$ & & & \\
\hline & BnaDYDH_05_2010a & $0.93^{* *}$ & 0.90 & & \\
\hline & BnaWAIT_01_2010a & $0.99 * * * *$ & $0.99^{* * * *}$ & $0.92^{* * * *}$ & \\
\hline & BnaWAIT_01_2010b & $1.00^{* * * *}$ & $1.00^{* * * *}$ & $0.98^{* * * *}$ & $0.97^{* * * *}$ \\
\hline \multirow[t]{4}{*}{$\mathrm{C01}$} & BnaSGDH_03_2010a & $0.97^{* * * *}$ & & & \\
\hline & BnaDYDH_05_2010a & 1.00 & $0.93^{* *}$ & & \\
\hline & BnaWAIT_01_2010a & $0.99^{* * * *}$ & $0.96^{* * * *}$ & $0.97^{* * * *}$ & \\
\hline & BnaWAIT_01_2010b & $1.00^{* * * *}$ & $0.99^{* * * *}$ & $1.00^{* * * *}$ & $0.97^{* * * *}$ \\
\hline \multirow[t]{4}{*}{$\mathrm{CO2}$} & BnaSGDH_03_2010a & $0.64^{*}$ & & & \\
\hline & BnaDYDH_05_2010a & 0.70 & 1.00 & & \\
\hline & BnaWAIT_01_2010a & $0.99 * * * *$ & $0.88^{* * * *}$ & $0.99^{* * * *}$ & \\
\hline & BnaWAIT_01_2010b & $1.00^{* * * *}$ & $0.99^{* * * *}$ & $1.00^{* * * *}$ & $0.96^{* * * *}$ \\
\hline
\end{tabular}


Table 3 Spearman's rank correlation $(r)$ of the marker order for the comparison among the three population-specific B. napus maps, BnaSNDH_05_2010a, BnaSGDH_03_2010a and BnaDYDH_05_2010a, comparison between each of the three population-specific maps and each of the two integrated maps, BnaWAIT_01_2010a generated by JoinMap and BnaWAIT_01_2010b generated by MergeMap and comparison between the two integrated maps, BnaWAIT_01_2010a and BnaWAIT_01_2010b (Continued)

\begin{tabular}{|c|c|c|c|c|c|}
\hline \multirow[t]{4}{*}{$\mathrm{CO3}$} & BnaSGDH_03_2010a & $0.91^{* * * *}$ & & & \\
\hline & BnaDYDH_05_2010a & 0.66 & $0.93^{* * * *}$ & & \\
\hline & BnaWAIT_01_2010a & $0.96^{* * * *}$ & $0.96^{* * * *}$ & $0.95^{* * * *}$ & \\
\hline & BnaWAIT_01_2010b & $0.98^{* * * *}$ & $1.00^{* * * *}$ & $1.00^{* * * *}$ & $0.96^{* * * *}$ \\
\hline \multirow[t]{4}{*}{$\mathrm{CO4}$} & BnaSGDH_03_2010a & $0.86^{* * * *}$ & & & \\
\hline & BnaDYDH_05_2010a & 1.00 & $0.96^{* *}$ & & \\
\hline & BnaWAIT_01_2010a & $0.89^{* * * *}$ & $0.96^{* * * *}$ & $0.99 * * * *$ & \\
\hline & BnaWAIT_01_2010b & $0.99 * * * *$ & $0.98^{* * * *}$ & $1.00^{* * * *}$ & $0.92^{* * * *}$ \\
\hline \multirow[t]{4}{*}{$\mathrm{CO5}$} & BnaSGDH_03_2010a & $0.95^{* * * *}$ & & & \\
\hline & BnaDYDH_05_2010a & 0.70 & $1.00^{* * *}$ & & \\
\hline & BnaWAIT_01_2010a & $0.99 * * * *$ & $0.98^{* * * *}$ & $0.47^{* * *}$ & \\
\hline & BnaWAIT_01_2010b & $1.00^{* * * *}$ & $1.00^{* * * *}$ & $1.00^{* * * *}$ & $0.81^{* * * *}$ \\
\hline \multirow[t]{4}{*}{$\mathrm{C06}$} & BnaSGDH_03_2010a & $0.69^{*}$ & & & \\
\hline & BnaDYDH_05_2010a & 1.00 & 1.00 & & \\
\hline & BnaWAIT_01_2010a & $0.63^{* * * *}$ & $0.82^{* * * *}$ & $1.00^{* * * *}$ & \\
\hline & BnaWAIT_01_2010b & $0.97^{* * * *}$ & $0.97^{* * * *}$ & $1.00^{* * * *}$ & $0.72^{* * * *}$ \\
\hline \multirow[t]{4}{*}{$\mathrm{CO7}$} & BnaSGDH_03_2010a & $0.91^{* * * *}$ & & & \\
\hline & BnaDYDH_05_2010a & 1.00 & $0.78^{* *}$ & & \\
\hline & BnaWAIT_01_2010a & $0.99 * * * *$ & $0.99 * * * *$ & $0.99 * * * *$ & \\
\hline & BnaWAIT_01_2010b & $1.00^{* * * *}$ & $0.98^{* * * *}$ & $1.00^{* * * *}$ & $0.99 * * * *$ \\
\hline \multirow[t]{4}{*}{$\mathrm{C08}$} & BnaSGDH_03_2010a & $0.96^{* * * *}$ & & & \\
\hline & BnaDYDH_05_2010a & 1.00 & 0.70 & & \\
\hline & BnaWAIT_01_2010a & $0.92^{* * * *}$ & $0.96^{* * * *}$ & $1.00 * * * *$ & \\
\hline & BnaWAIT_01_2010b & $1.00^{* * * *}$ & $1.00^{* * * *}$ & $1.00^{* * * *}$ & $0.94^{* * * *}$ \\
\hline \multirow[t]{4}{*}{$\mathrm{C09}$} & BnaSGDH_03_2010a & $0.94^{* * * *}$ & & & \\
\hline & BnaDYDH_05_2010a & 1.00 & 1.00 & & \\
\hline & BnaWAIT_01_2010a & $0.99 * * * *$ & $0.99^{* * * *}$ & $0.98^{* * * *}$ & \\
\hline & BnaWAIT_01_2010b & $0.99 * * * *$ & $1.00^{* * * *}$ & $1.00 * * * *$ & $0.99^{* * * *}$ \\
\hline
\end{tabular}

The level of significance is shown by asterisks. Significance is shown as: ${ }^{*}<0.05 ;{ }^{* *}<0.01 ;{ }^{* *}<0.001 ;{ }^{* * *}<0.0001$.

BnaWAIT_01_2010a integrated map $(2,114 / 5,162)$ displayed homology to Arabidopsis, representing 39.2\% of all mapped loci in the BnaWAIT_01_2010a integrated map. All the information of high-scoring segment pairs (HSP) and their relations to the BnaWAIT_01_2010a integrated map are available in Additional File 3.

For the identification of collinearity blocks conserved between B. napus and Arabidopsis genomes, we employed similar criteria to Parkin et al. [7]. A conserved block was defined as being supported by at least four homologous loci with at least one shared locus within every $5 \mathrm{cM}$ in $B$. napus, and at least one shared locus within every $1 \mathrm{Mb}$ in Arabidopsis. Based on these criteria, we detected 103 collinearity blocks in the B. napus genome in relation to Arabidopsis, of which 45 showed a significant correlation in the marker order for shared loci between $B$. napus and Arabidopsis ( $p<0.05$, Additional File 7$)$. Each block contained on average 12 shared loci, and had an average length of $10.0 \mathrm{cM}$ in B. napus and $2.8 \mathrm{Mb}$ in Arabidopsis. The blocks represent $1,026 \mathrm{cM}$ of the $B$. napus integrated map (57.3\% of the mapped length) and $87.6 \mathrm{Mb}(74.2 \%)$ of the Arabidopsis genome sequence. It appeared that the mapped genetic lengths of conserved blocks were significantly positively correlated with the aligned physical chromosomal lengths of Arabidopsis across all blocks (Spearman's correlation $r=0.64, p=2.84 \mathrm{e}-13$ ). The longest conserved block in terms of genetic length was BnaWAIT_A_26 in A05 with the genetic length of $49.1 \mathrm{cM}$ (49.0\% of the LG length), supported by 30 shared loci. The block with the highest number of shared loci was BnaWAIT_C_49 in C09 (44). The longest block in terms of aligned physical length was BnaWAIT_A_20 in A04 which was aligned to $10.9 \mathrm{Mb}$ of Arabidopsis chromosome 2 (Arabidopsis blocks C2B and C2C). 
Consistent with previous findings, we also found evidence of inversions and internal duplications within LGs relative to Arabidopsis (Additional File 7). In A07, the blocks arising from chromosomal segmental duplications, BnaWAIT_A_38 and BnaWAIT_A_39, were adjacent to each other with reversed orientation, consistent with an inverted duplication block (IDB sensu [56]). This has also been observed in the homeologous chromosome C06 in Brassica oleracea [57,58] and B. napus [56]. There was also evidence that some blocks overlapped with each other, and that some blocks were nested within other blocks. The overlapping genetic distances between blocks (also including blocks which were nested within another block) varied from $0.5 \mathrm{cM}$ up to $10.1 \mathrm{cM}$ within LGs (Additional File 7).

\section{Genome duplication within the Brassica genome}

The BnaWAIT_01_2010a integrated map enabled us to investigate the global genome organization of $B$. napus relative to the Arabidopsis genome. Consistent with previous observations [7] there were between 5 and 8 conserved collinearity blocks distributed across the 19 B. napus chromosomes for each Arabidopsis block (Figure 3). It appeared that the Arabidopsis blocks adopted in the BnaSNDH_03_2005a map [7] were sufficient to describe the pattern of genome triplication in the BnaWAIT_01_2010a map. There was stronger evidence for genome triplication within Brassica for some Arabidopsis blocks compared with others, supported by a higher number of shared loci and longer continuous collinearity block between the two genera across LGs (e. g. blocks C1A, C1B, C2C, C3A, C3 D, C4B, C5A and C5E). Arabidopsis chromosomal regions having at least 5 continuous homologous copies within B. napus covered approximately $80 \%$ of the Arabidopsis genome (Figure 3).

\section{Comparative mapping of $B$. napus and $B$. rapa A genome}

The BnaWAIT_01_2010a integrated map also enabled us to investigate the A genome evolutionary dynamics since the hybridization with the $\mathrm{C}$ genome. We mapped all sequence tagged markers used in the BnaWAIT_01_2010a integrated map onto the B. rapa A genome anchored scaffolds (The Brassica rapa Genome Sequencing Project Consortium [59]) for each chromosome, and compared the marker order of genetic distances (cM) with that of physical distances $(\mathrm{Mb})$ using dot-plots and rank correlation.

Marker order was globally conserved between the $B$. napus A genome integrated map and the B. rapa $\mathrm{A}$ genome anchored scaffolds across all 10 chromosomes despite some local discrepancies (Figure 4, Table 4). In A03, the correlation between the genetic length and the physical length appeared to be almost linear across the entire chromosome. The poorest correspondence between genetic and physical maps was found in A08 (Spearman's correlation $r=0.65, p<0.0001)$. In some regions of the genome, the local order was clearly shown to be inconsistent between the integrated genetic map of $B$. napus A genome and the $B$. rapa genome scaffolds, such as the top section of LG A08 (0 - 8 cM)

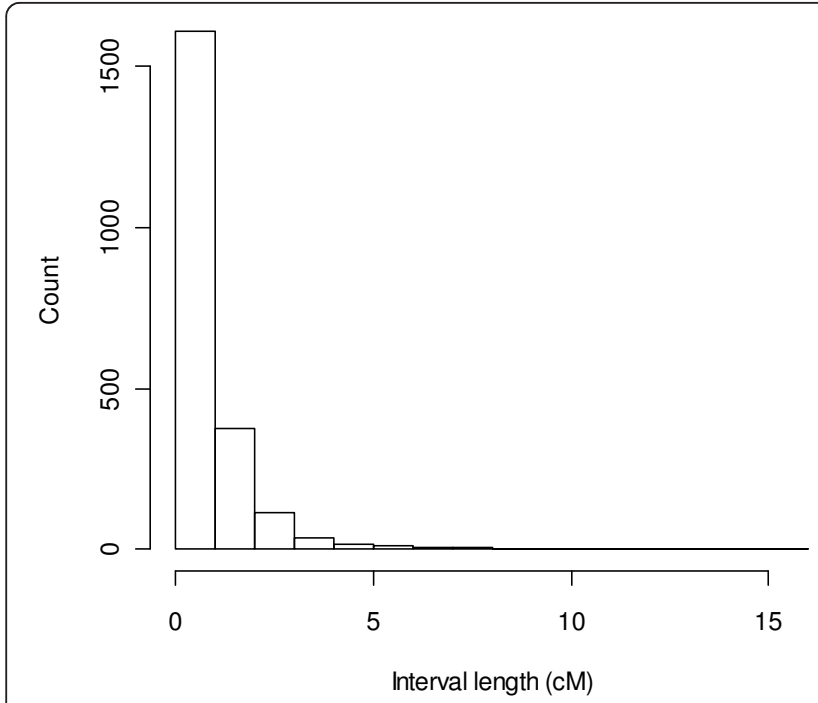

(a)

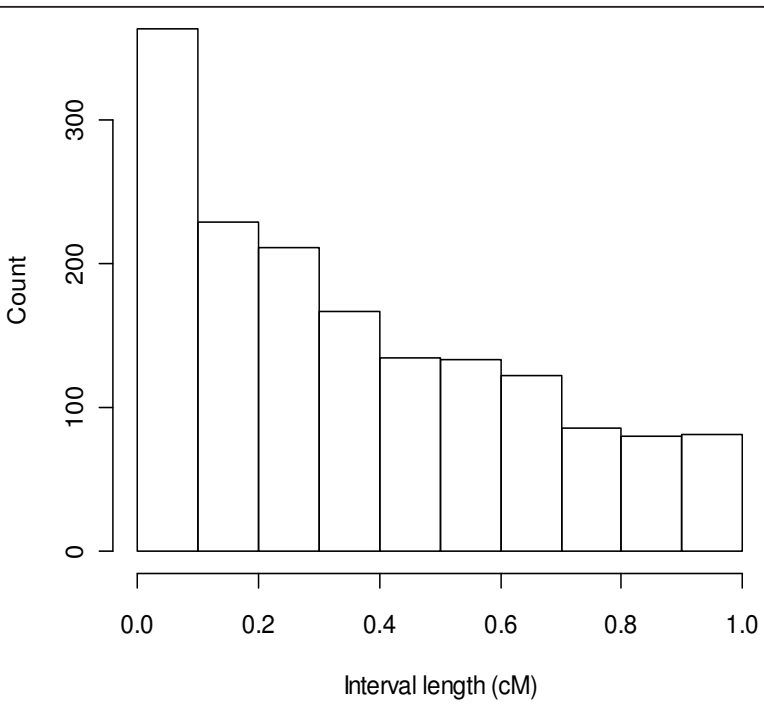

(b)

Figure 1 The distribution of map intervals for the BnaWAIT_01_2010a integrated map generated by JoinMap. It is highly skewed with an abundance of shorter distances. a) shows the distribution from 0-1 cM to >10 cM sections. b) shows the further partition of interval length distribution within the 0-1 cM section. 


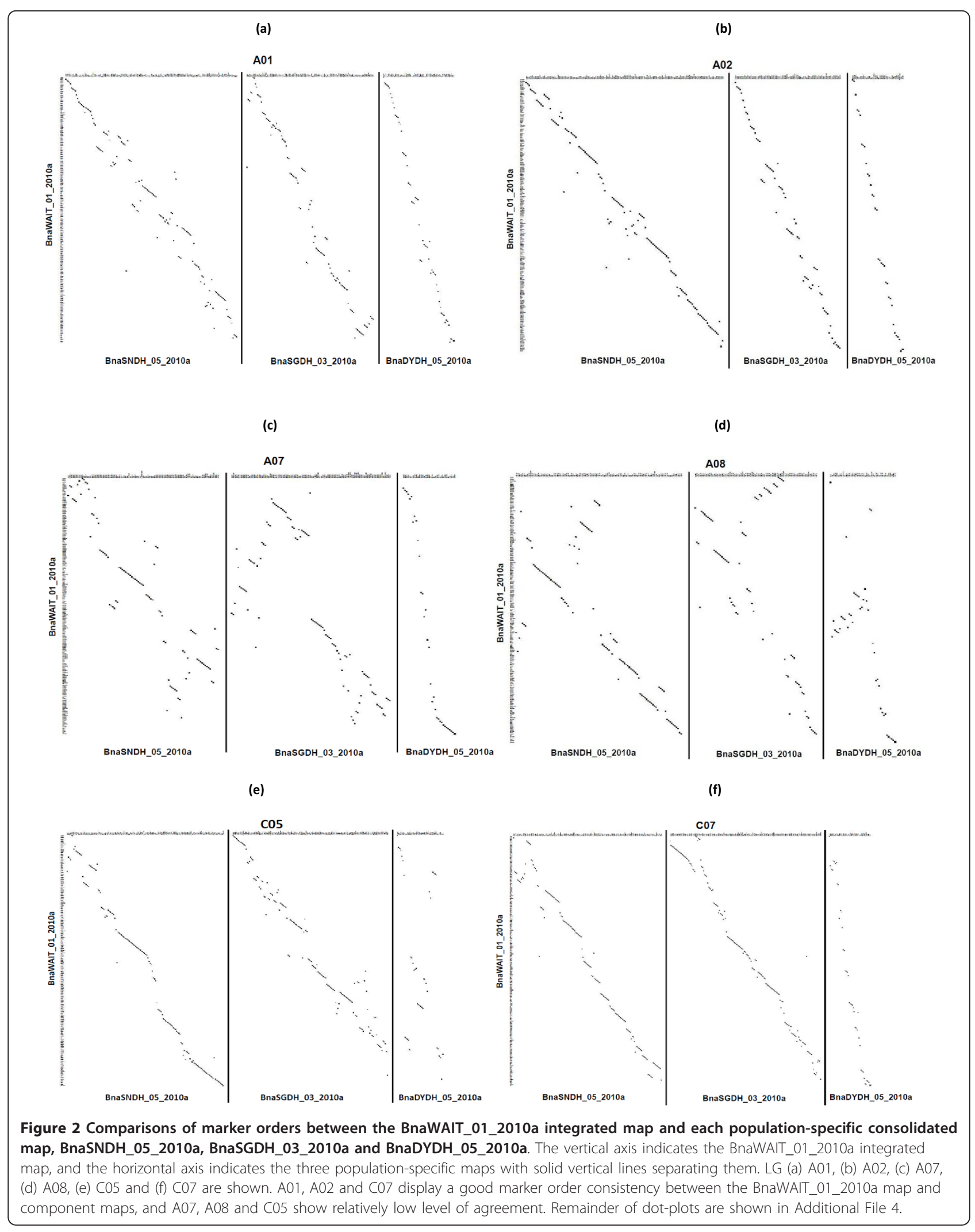


(Figure 4). The local correlation between the genetic distance and the physical distance in this region $(r=-0.62$, $p<0.05)$ appeared to be of opposite sign to the global correlation for the whole chromosome. This appeared to result from the fact that more than half of the loci in this region were physically mapped to the bottom of the chromosome (10 - $17 \mathrm{Mb})$. Moreover, this region of $8 \mathrm{cM}(10.8 \%$ of the genetic length of A08) covered $\sim 15 \mathrm{Mb}$ of physical length ( $75 \%$ of the whole chromosome physical length). Interestingly, A08 also had the lowest correlations of marker order between populationspecific maps and the BnaWAIT_01_2010a integrated map (Table 3). In A05, both ends of the chromosome $(0-5 \mathrm{Mb}$ and $20-25 \mathrm{Mb}$ ) together corresponded to $\sim 90 \%$ of the genetic length. We further investigated two additional LGs, A07 and A09, with relatively low correlations of marker order between population-specific maps and the integrated map, compared with other LGs in the A genome (Table 3). Both LGs also showed relatively lower correlations in the marker order between the integrated genetic map and the physical $B$. rapa genome sequences $(r=0.80$ for A07, $r=0.86$ for A09, Table 4).

We then carried out the comparison of marker order between each population-specific consolidated map and the $B$. rapa genome scaffolds using rank correlation. It showed that for most of the LGs, the correlation coefficient was $>0.85$ for all three individual population-specific maps in relation to the physical $B$. rapa scaffolds. This correlation was relatively weaker for LGs A07, A08 and A09 (Table 4). Interestingly, for A08, both BnaSNDH_05_2010a and BnaSGDH_03_2010a maps showed very high correlations, but the BnaDYDH_05_2010a showed a very poor correlation with the physical B. rapa scaffolds (Table 4). The BnaSGDH_03_2010a map also showed a similar pattern of discrepancy against the physical $B$. rapa sequence in A09. The marker order discrepancies between some population-specific maps and the physical $B$. rapa sequence for some LGs may derive from the genome structural variation (deletion, inversion and translocation) between populations.

\section{Discussion}

Over the past two decades more than 20 substantial genetic maps have been published for different Brassica species but little concerted efforts has been made to align maps from different populations. We have collated both published and previously unpublished genomewide genotype data for sequence-tagged RFLP and SSR markers scored on three widely used Brassica napus



Figure 3 Genome duplication within the $\boldsymbol{B}$. napus genome relative to five Arabidopsis chromosomes. Each dot represents an alignment between a genetic marker of B. napus and its homology BLAST hit within Arabidopsis chromosomes. i) the Arabidopsis blocks used in Parkin et al. [7], ii) the ancient karyotype (AK) blocks from Schranz et al. [83] are shown alongside the dot-plots aligned to their Arabidopsis chromosomal positions. 


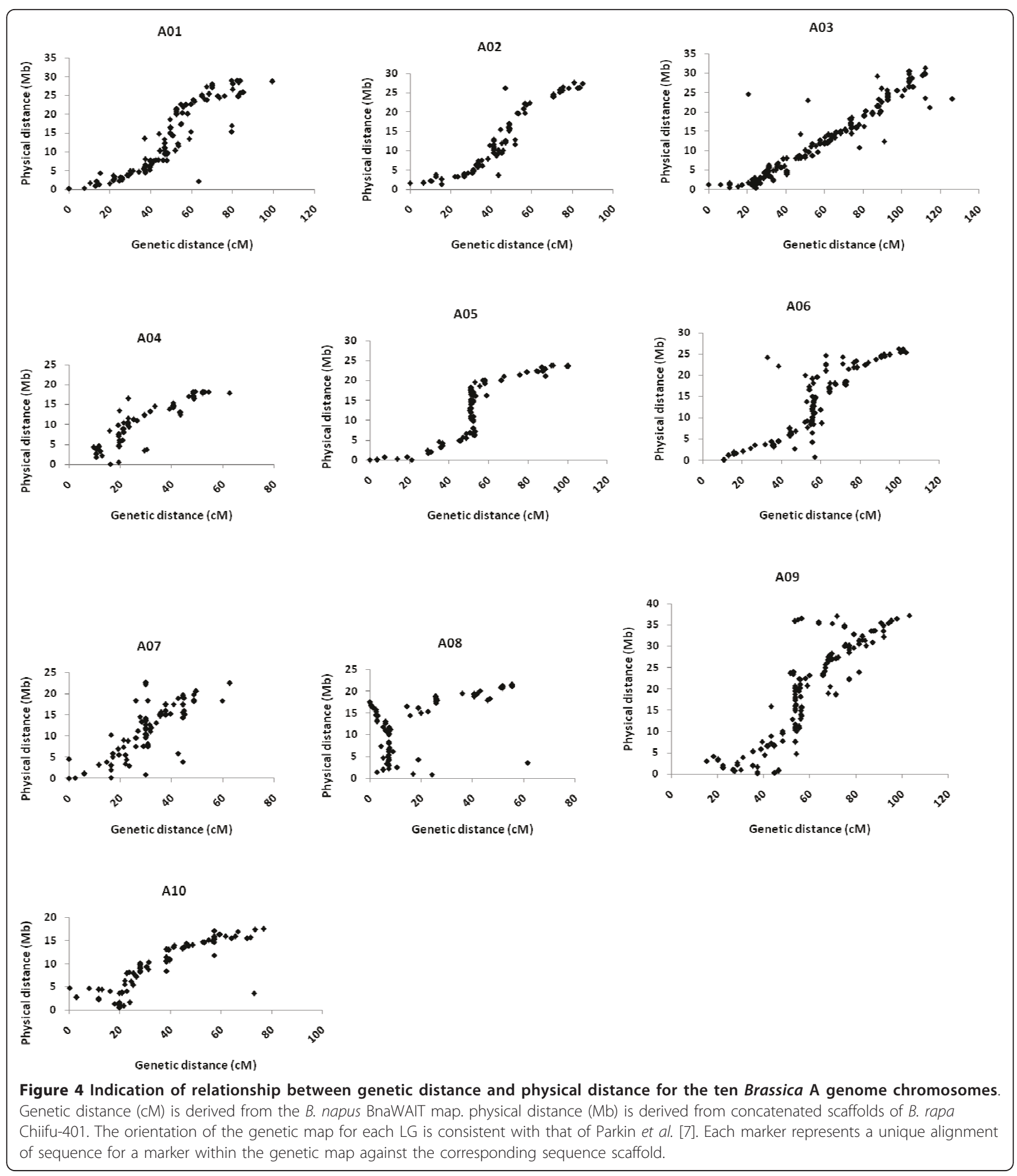

populations of doubled haploid lines (BnaSNDH, BnaSGDH and BnaDYDH).

Constituent genotype matrices for each of the 19 linkage groups (LGs) were first combined to generate a consolidated genetic map for each population. Integration of component genetic maps involved selection either of bridge markers shared between populations or of markers with the highest information content to represent each unique mapping locus (bin). The skeleton bin maps for the three populations were then combined to 
Table 4 Spearman's rank correlation $(r)$ of the marker order of the integrated map BnaWAIT_01_2010a, the three population-specific maps, BnaSNDH_05_2010a, BnaSGDH_03_2010a and BnaDYDH_05_2010a, against the physical B. rapa A genome scaffolds.

\begin{tabular}{|c|c|c|c|c|}
\hline LG & BnaSNDH_05_2010a & BnaSGDH_03_2010a & BnaDYDH_05_2010a & BnaWAIT_01_2010a \\
\hline $\mathrm{A} 01$ & $0.97^{* * * *}$ & $0.95^{* * * *}$ & $0.93^{* * * *}$ & $0.96^{* * * *}$ \\
\hline $\mathrm{A} 02$ & $0.96^{* * * *}$ & $0.98^{* * * *}$ & $0.84^{* * * *}$ & $0.97^{* * * *}$ \\
\hline A03 & $0.97^{* * * *}$ & $0.96^{* * * *}$ & $0.92^{* * * *}$ & $0.97^{* * * *}$ \\
\hline A04 & $0.95^{* * * *}$ & $0.92^{* * * *}$ & $0.94^{* * * *}$ & $0.92^{* * * *}$ \\
\hline A05 & $0.95^{* * * *}$ & $0.95^{* * * *}$ & $0.91^{* * * *}$ & $0.91^{* * * *}$ \\
\hline A06 & $0.91^{* * * *}$ & $0.86^{* * * *}$ & $0.92^{* * * *}$ & $0.92^{* * * *}$ \\
\hline A07 & $0.79^{* * * *}$ & $0.72^{* * * *}$ & 0.87 & $0.80^{* * * *}$ \\
\hline A08 & $0.96^{* * * *}$ & $0.98^{* * * *}$ & 0.23 & $0.65^{* * *}$ \\
\hline A09 & $0.88^{* * * *}$ & $0.64^{* * * *}$ & $0.91^{* * * *}$ & $0.86^{* * * *}$ \\
\hline A10 & $0.95^{* * * *}$ & $0.97^{* * * *}$ & $0.76^{* * * *}$ & $0.95^{* * * *}$ \\
\hline
\end{tabular}

The level of significance is shown by asterisks. Significance is shown as: ${ }^{*}<0.05 ;{ }^{* *}<0.01 ;{ }^{* * *}<0.001 ;{ }^{* * *}<0.0001$.

generate an integrated map for each LG, comparing two different approaches, one encapsulated in JoinMap and the other in MergeMap. JoinMap made use of the full set of available genotype scores whilst MergeMap made use of the marker orders and cM distances of the component maps. Although the performance of MergeMap depends on the quality and accuracy of marker order within component maps, this approach has been shown to outperform JoinMap both in terms of accuracy and running time based on simulated data [50], and has been used successfully to construct integrated maps in barley [60] and cowpea [41].

In the present study, a relatively low proportion of marker loci $(20.2 \%)$ were common to at least two populations. This may not provide sufficient information to overcome a few cases of uncertainty in locus order that were present in the component maps (e.g., between BnaSGDH and BnaDYDH for A04 and A07, and between BnaDYDH and the other two maps for C06, Additional File 1). However, for the purpose of map alignment/integration, the consistency of order among common markers between individual maps appeared to be more important than simply the number of shared loci. Our results demonstrate that the marker order was generally well conserved (i.e., a high level of collinearity) in the component maps, which provided a good foundation for the subsequent map integration analyses. Indeed, both JoinMap and MergeMap generated integrated maps with good consistency in marker order (measured by Spearman's rank correlation coefficient $r$ ) compared with component population-specific maps for most LGs (JoinMap, $r>0.90$ for all three pairwise comparisons for 11 LGs; MergeMap, $r>0.95$ for the three pairwise comparisons for all 19 LGs). MergeMap improved the marker order consistency for some LGs where JoinMap performed relatively poorly (e.g., A07, A08, C05 and C06).
There may be several reasons why JoinMap appeared to perform relatively poorly for some LGs. This includes the low number of shared 'bridge' markers between component maps which may hide underlying conflicts in genotype ordering that is accessible to JoinMap and not used by MergeMap. Resolving such conflicts in marker order is relatively straightforward for MergeMap as it makes use of directed acyclic graphs (DAG) to generate a single directed graph according to their shared vertices. Any ordering conflict between individual maps resulted in cycles in the combined graph. MergeMap then resolves the cycles (conflicts) by identifying and eliminating a small number of marker occurrences from some of the maps after weighting marker order differences. MergeMap only requires the marker order and cM distances of the component maps rather than the data of original genotype scores of individual populations. Thus it may be possible for consistent errors in the marker order or interval lengths in a majority of component maps to be incorporated into the integrated maps. However, in this study we can be reasonably confident that the component maps were a reliable representation of $B$. napus chromosomes, since the maps from independent populations and in different laboratories generated similar marker order. MergeMap was therefore expected to produce a relatively reliable marker order in the integrated map. In contrast, JoinMap is constrained by its need to resolve a consistent marker order in the integrated map based on a limited number of mean recombination frequencies and combined LOD scores. For both methods, when the degree of marker order inconsistency increases between individual maps, the performance becomes relatively inferior. Establishing the thresholds of such inconsistencies will be important for more extensive map integration where larger numbers of maps and/or reduced numbers of bridge markers are available. 
Furthermore, one should note that there would be always conflicting markers between/among different component maps to be merged (Table 4). These conflicts of marker orders could be derived from the genome structural variation (deletion, inversion and translocation) between populations for some LGs or mapping errors. Thus, low correlations between the integrated map and a particular population-specific map, along with good correlations between the integrated map and the other two component maps (Table 3 and 4), could be indications of genome rearrangements in one of the populations. Further investigation of the dot plots (Figure 2 and Addition File 4) may identify the event(s) which creates such marker order conflicts.

As part of the pre-processing of genotype data prior to map integration, we carried out a masking of genotype scores where single data points were eliminated where a single locus was flanked by a double crossover. This process provides more consistent genetic lengths for specific linkage groups, and more realistic lengths between adjacent crossovers that represent exchange of large chromosomal regions. This process may also eliminate some actual genetic exchanges. However, since these would be short they will have only a small effect on the final map. Following this procedure a degree of map inflation still remained compared with those published previously for BnaSNDH $[7,16]$ and BnaDYDH $[19,25]$, which is often encountered when large numbers of markers are employed due to the cumulative effect of the low background error rate. Any overestimation of genetic length is incorporated into integrated maps calculated by MergeMap. In contrast, JoinMap makes use of all available pairwise recombination frequencies and LOD scores, and so LG lengths were closer to expectation and appeared more reliable, with good agreement with previously published component maps. In addition, JoinMap was also able to resolve a greater number of unique marker loci across all LGs, increasing the number of loci by $22.8 \%$ compared with MergeMap.

The heuristic method employed in MergeMap greatly enhances the speed of map integration compared with the regression mapping algorithm employed in JoinMap, especially where large genotype matrices are used. Indeed JoinMap is limited by the matrix size for dense maps, and so the problem needs to be broken down into sub-problems, either by bin mapping as we have done here, or by taking overlapping sub-sections of LGs, which does not provide an ideal solution. Pragmatically, where accurate estimates of genetic distances are not the priority, MergeMap provides a rapid and relatively reliable solution, especially where component maps have been generated with consistently low error rates for marker scores. The MergeMap algorithm has been successfully applied for map integration where either a large number of genetic markers are involved, such as high-throughput SNP genotyping [60], or where genotyping data were not available for many published genetic maps [61]. However, JoinMap still performed well in map integration based on our map construction procedure for the three $B$. napus $\mathrm{DH}$ populations.

Overall, the BnaWAIT_01_2010a integrated map generated by the JoinMap method included 5,162 markers, compared with 1,317 markers in the previous reference BnaSNDH map of Parkin et al. [7] and 866 markers in the BnaDYDH map reported by Delourme et al. [25]. This increased the marker density by 3.3 and 5.8 fold, respectively. Furthermore, the nine LGs representing C genome chromosomes contain $11.6 \%$ more markers and $11.8 \%$ more loci than the ten LGs representing A genome chromosomes in the BnaWAIT_01_2010a map. This is in close agreement with the estimated $16 \%$ larger size of the $\mathrm{C}$ genome $[53,54]$.

The BnaWAIT_01_2010a integrated map enabled us to test existing models of collinearity between Arabidopsis and Brassica. This analysis was based on twice as many markers where sequence similarity to Arabidopsis could be identified, compared with the BnaSNDH map of Parkin et al. [7]. We identified 103 conserved colinearity blocks in $B$. napus relative to Arabidopsis. These corresponded to almost all $97 \mathrm{~B}$. napus blocks reported in the BnaSNDH_03_2005a map, although we did not resolve 17 short blocks previously identified based solely on RFLP markers [7]. Although the same homology hits were identified between the Arabidopsis genome and 50 RFLPs within these 17 short blocks, the criteria to define a collinearity block (i.e., four homologous loci with at least one shared locus within every $5 \mathrm{cM}$ in B. napus and at least one shared locus within every 1 $\mathrm{Mb}$ in Arabidopsis) were not met in our study. Moreover, these short blocks only represented $<5.0 \%$ of the total mapped length of the BnaSNDH_03_2005a map. Five previously unreported collinearity blocks were identified in our study. However, these new blocks covered only $14.5 \mathrm{cM}$ of genetic length in total, aligned to 7.0 $\mathrm{Mb}$ in Arabidopsis chromosomes 3 and 5. We further established that the synteny order of the 48 collinearity blocks within the A genome of B. napus in BnaWAIT_01_2010a is essentially the same as that established in B. juncea based on intron polymorphism (IP) markers [10]. This indicates that synteny order is highly conserved in the A genomes of B. juncea and B. napus.

We attempted to align 3,837 primer sequence pairs for the SSR markers to the Arabidopsis chromosomes to identify homology with the resultant target 'virtual PCR product' of primers. However, $<2 \%$ of the primer pairs had homology in Arabidopsis, of which only 50\% agreed with those identified using the corresponding SSR clone sequences. This suggests that future comparative studies 
within the Brassicaceae based solely on SSR primer sequences are unlikely to provide useful information where sequences have diverged over similar time scales.

The increased marker density provided by the integrated map is a valuable resource that increases the availability of markers in regions of interest, thus assisting in fine mapping. It also provides additional information for comparative mapping studies, e.g., to detect potential genome rearrangements in some populations. Furthermore, the increase in density of sequence tagged markers and availability of draft genome sequence scaffolds, enabled us to carry out a preliminary investigation of the relationship between genetic and physical distances in the Brassica A genome. This indicated that the chiasmata were not evenly distributed within chromosomes, and that there was considerable variation in the pattern of crossovers between chromosomes. Many studies have suggested the distribution of meiotic crossover events along chromosomes in plants and other species is non-random [62-66]. Non-random distributions of crossover rates have been reported to be correlated with several chromosomal features, including chromosome size, gene density, presence of transposable elements or heterochromatin, and distance to centromeres [67-72]. However, the underlying mechanisms affecting chiasmata distribution may be taxa specific [73], and so it is important to establish any relationships within or between Brassica chromosomes and species. Within the $C$ genome of $B$. oleracea, a clear difference in relationship between genetic and physical distances has been established for IDBs on C6 [58]. The analysis we have carried out is preliminary and any mechanistic understanding will require more complete genome sequence scaffold data that include details of the distribution of repetitive DNA and of degree of chromatin condensation. In addition, it may be necessary to select additional markers that represent the full length of individual chromosomes. Based on complete genome sequence data, Drouaud et al. [74] have been able to resolve details of non-random distribution of chiasmata in relation to heterochromatic knobs and other chromosomal feature on Arabidopsis chromosome 4. Access to larger populations and more reliable sequence-tagged mapping methods (e. g., high-density SNP mapping) are likely to increase the resolution and understanding of the basis of variation in recombination frequency in Brassica.

We also attempted to anchor the remainder of the unanchored A genome scaffolds onto LGs based on the B. napus integrated map, and this anchored three additional scaffolds. Given the genome structure of Brassica, some scaffolds will be in repeat-rich or duplication regions, and thus it is difficult to resolve the LG assignments.

\section{Conclusions}

In summary, we have generated a comprehensive integrated map for the $B$. napus genome, which includes 5,162 genetic markers mapped onto 2,196 loci, with a total genetic length of $1,792 \mathrm{cM}$. The map density of one locus every $0.82 \mathrm{cM}$, corresponding to $515 \mathrm{Kbp}$, increases by at least three-fold the marker density within the original maps. The BnaWAIT map thus provides access to additional informative markers, which will assist in resolution and fine mapping of QTL regions, as well as facilitating marker-assisted introgression and selection in Brassica crops. Our map integration pipeline is readily applied to map integration studies for other genera. The population-specific consolidated maps and the integrated maps are publicly available http:// www.cropstoredb.org/brassica and provide a valuable resource in fine mapping and comparative mapping studies for Brassica research.

\section{Methods}

\section{Component maps, genetic markers and genotype data}

Three extensively studied Brassica napus mapping populations of doubled haploid (DH) lines, BnaSNDH, BnaDYDH and BnaSGDH (Additional File 8) were used to construct integrated maps. The BnaSNDH $[7,16]$ and BnaDYDH $[19,25]$ populations have been described previously. The BnaSGDH population was derived from an F1 generated from a cross between PSA12 (a resynthesized $B$. napus line generated from a cross between $B$. oleracea A12DHd and B. rapa Parkland Sunshine hybrid) and DH12075 (a DH line derived from a Westar $\times$ Cresor cross). All the mapping data (e.g., genetic maps and genotyping scoring matrices) of the three $\mathrm{DH}$ populations for the 19 linkage groups (LGs) have been collated and curated into the CropStoreDB database that provides a registry of data relating to Brassica genetics http://www. cropstoredb.org/brassica.

Assignment of marker loci to existing linkage groups was already available for a subset of previously published component maps (Additional File 8), BnaSN DH_02_2004a [20], BnaSNDH_03_2005a [7], BnaDYDH_01_2001a [19] and BnaDYDH_03_2008a [25]. These had been calculated using Mapmaker v3.0 [75,76], with LGs assigned at a threshold LOD score of $>=4.0$. Similarly a component linkage map had been developed for BnaSGDH using a core set of RFLP markers. Additional SSR genotyping data for BnaSNDH and BnaSGDH (Additional File 8) were provisionally assigned to existing LGs by string-matching and linkage map distances were confirmed and calculated using Mapmaker v3.0. For each population, the composite sets of genotype data were pooled to generate a single matrix for each of the 19 linkage groups (LGs). Missing values (notated as "-") were assigned where a marker had not 
been genotyped for a particular individual line. Where scoring strings had been collated from more than one source for the same marker in the same population, the set containing the greater number of genotype scores was retained.

\section{Map construction}

The overall process of map integration is outlined in Figure 5. Each merged scoring matrix was analysed using JoinMap version 4.0 [48]. Linked loci were grouped with a LOD grouping threshold ranging from 3.0 to 5.0. Locus order within the LOD grouping was generated for each LG using the maximum likelihood (ML) algorithm with default parameters. The Kosambi map function was used to estimate genetic distances. Following initial ordering, the genotype matrix for each LG was investigated and data points were eliminated where a single locus was flanked by a double crossover. The modified genotype matrix for each LG was then imported again into JoinMap for linkage analysis using the same grouping and ordering algorithm and parameters. This procedure reduced the linkage map length for each LG in the integrated map by an average of $>100 \mathrm{cM}$. Linkage groups were orientated consistent with Parkin et al. [7].

Prior to construction of an integrated map, population-specific bin maps were generated for each linkage group using a modification of the method described by Howad et al. [77]. A bin was defined where a unique map position was assigned. Thus, a bin may contain just one marker or more than one marker up to $\sim 20$ markers in our maps. Moreover, markers within $<1 \mathrm{cM}$ were also assigned to the same bin. A bin continued until a new map position was $\geq 1 \mathrm{cM}$ distance from the first map position of the bin. The next bin would then start from the new map position. For each bin, a single genetic marker was selected that either provided a bridge to at least one other population-specific map, or maximised the information content with the maximum number of genotype/line scores. Following map calculation based on these binned genotype matrices, the residual markers were re-introduced and assigned to their bin positions.

We compared two different approaches for map integration, based on MergeMap and JoinMap procedures. In MergeMap [50], individual maps are first converted to directed acyclic graphs (DAG), which are merged into a consensus graph on the basis of their shared vertices. MergeMap then attempts to resolve conflicts among individual maps by deleting a minimum set of marker occurrences. The result of the conflict-resolution step is a consensus DAG, which is then simplified and linearised to produce the final consensus map.
JoinMap 4.0 [48] was used to generate pairwise recombination frequencies and LOD scores for the selected sets of representative loci for each linkage group, which were then combined into a single group node in the navigation tree. Within JoinMap the "Combine Groups for Map Integration" function carries out map calculations based on mean recombination frequencies and combined LOD scores [48]. The regression mapping algorithm was used and the LG lengths for the consensus map of all the representative markers were calculated. Values for the "jump" threshold ranged from 4.0 to 6.0. When more than $\sim 150$ markers are present, JoinMap is limited by computational constraints, as its computation time is the fourth power of the number of markers.

The final stage involved local rearrangement of marker order, where there was evidence of physical proximity based on homology to sequences co-located on contiguous stretches of DNA. Since this was primarily available for Brassica rapa BACs http://www.brassica.info/ resources.php, this evidence was strongly weighted to A genome LGs. In the absence of evidence from recombination (i.e., within the same map bins), the local order was sorted with the assumption of collinearity with Arabidopsis, based on the order of orthologous gene models and the previously described internal synteny block structure [7].

\section{Homology search between Arabidopisis and Brassica rapa}

For each set of markers (Additional File 6 and 8) we identified the corresponding DNA sequences. This information has been collated and curated with the CropStoreDB and SeqStoreDB databases http://www.cropstoredb.org. SeqStoreDB contains records of all publicly available Brassica sequences released in GenBank, together with clone and primer sequences from many public and proprietary sources. This enables unambiguous management of sequence collections of query and target sequences, with explicit dataset versioning and recording of data provenance. The sequences associated with each set of genetic markers were used as queries in homology searches against the Arabidopsis thaliana pseudo-chromosomes (TAIR9 release, ftp://ftp.arabidopsis.org/home/tair/ Sequences/), and against 1,089 sequenced B. rapa BACs available in NCBI GenBank (date version: 01/12/09). In addition, we were kindly provided with pre-publication access to 192 B. rapa Chiifu-401 genome scaffolds (255.9 $\mathrm{Mb}$, representing $90 \%$ of the assembled sequences) by Xiaowu Wang, IVF-CAAS, Beijing. These scaffolds have been analysed and incorporated into the Brassica rapa Genome Sequencing Project Consortium [59]. These Brassica A genome scaffolds had been assigned to chromosomes based on integration of information from several 


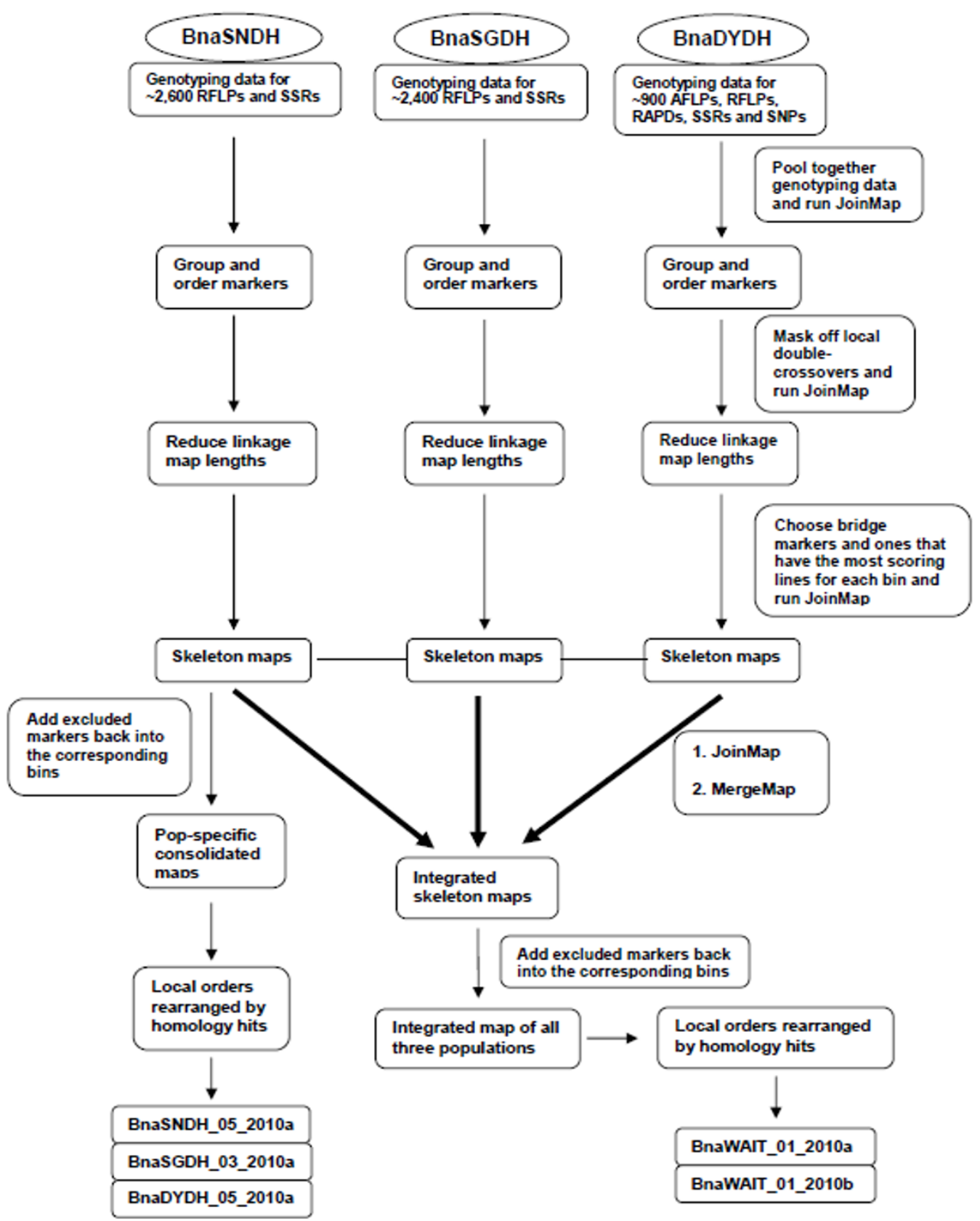

Figure 5 Flow diagram indicating the process of the genetic map integration for three B. napus DH populations, BnaSNDH, BnaSGDH and BnaDYDH

different B. rapa genetic maps including BraCKDH [78], BraJWF3 [79] and BraVCS_DH http://www.brassica-rapa. org, as well as a newly constructed map for the BraRCZ16_DH population based on 86 SSRs and 403 InDel markers developed directly from the scaffold sequences. Where scaffolds could not be assigned and orientated with respect to Brassica A genome chromosomes by genetic markers, provisional locations were assigned based on location within collinearity blocks relative to Arabidopsis (The Brassica rapa Genome Sequencing Project Consortium [59]).

For RFLP probes, homology searches used the TeraBLAST algorithm on a TimeLogic ${ }^{\mathrm{R}}$ solutions DeCypher system http://www.timelogic.com/, with parameters: match $=1$, mismatch $=-3$, gap open penalty $=-5$, gap extension penalty $=-2$, word size $=11 \mathrm{bp}$, and low complexity sequences filtered. A fairly low expect value ( $E$-value) was used as the exclusion cutoff $(1 E-07)$. 
High-scoring alignment segments were then further excluded where (1) the sequence identity was less than $86 \%$ between Brassica and Arabidopsis (the average sequence identity over all aligned sequence pairs for RFLPs used in Parkin et al. [7]) and (2) alignment length was less than 100 consecutive nucleotides.

For the SSR markers, we used the whole clone sequences from which the original primer sequences had been designed. RepeatMasker http://www.repeatmasker. org/ was first used to mask simple repeats and interspersed repetitive elements from each SSR set. The algorithm of Cross_Match http://www.phrap.org was implemented and the Brassicaceae Repeat Database from TIGR plant repeat database http://plantrepeats.plantbiology.msu.edu/brassicaceae.html was used as the repeat library. Each masked SSR set was queried using the TeraBLAST algorithm against target database sequences, using parameters: match $=1$, mismatch $=-1$, gap open penalty $=$ -2 , gap extension penalty $=-2$, word size $=11 \mathrm{bp}$, with the dust filter on. The cutoff $E$-value of $1 E-03$ was used. We further excluded alignments where the sequence identity was less than $80 \%$ between Brassica and Arabidopsis and alignment length was less than 30 consecutive nucleotides. A similar approach has also been used to indentify homologous hits of microsatellite sequences between livestock species $[80,81]$. The sequence divergence cutoff value was increased to $90 \%$ for alignments between marker sequences derived from $B$. napus clones, and those of $B$. rapa $\mathrm{BAC}$ clones or genome scaffolds. This is a lower value than that suggested by the divergence between orthologous sequences of two stearoyl-ACP desaturase loci from the A genome of $B$. rapa and B. napus, which had $97.5 \% \pm 3.1 \%$ sequence identity [82].

Where available, we also used SSR primer sequences ( 20 bp in length) in pairs directly as query sequences to search for homologies against the $A$. thaliana pseudochromosomes, using the Tera-Probe algorithm http:// www.timelogic.com/teraprobe.html with both gapped alignment and query filter options off. We allowed at most one mismatch between each of the primer sequences and the homologous $A$. thaliana sequences. Alignments were only accepted where both sequences from a primer pair had hits to the same $A$. thaliana chromosome, with the orientation consistent with the original conformation in Brassica, and the distance between the hits was shorter than $1000 \mathrm{bp}$ and longer than $150 \mathrm{bp}$.

Homology search alignments were managed within the AlignStoreDB relational database. This enabled explicit and cumulative querying of result sets in the context of sets of markers located on specific linkage groups (managed within CropStoreDB). The relationships between the different databases are shown in Additional File 9.

The marker loci within the Brassica integrated map were compared with the chromosomal location of corresponding genes with the highest homology (in terms of bit scores) in the Arabidopsis genome and B. rapa genome scaffolds. Collinearity blocks were colour-coded according to the convention of Parkin et al. [7]. Positions of markers in the integrated maps are shown within each component map. We compared the marker order of the integrated map generated from the three populations and those of populationspecific maps for each LG using dot plots. A dot was generated using a combination of a Perl script and the "conditional formatting" function within Microsoft Excel, and highlighted by linking the horizontal position in one map and the vertical position in the other map for a shared marker between the two maps. Such dot plots can be applied to compare marker orders for any pair of maps where there are shared markers. We then calculated Spearman's rank correlation coefficients for marker orders between pairs of maps.

\section{Additional material}

\begin{abstract}
Additional file 1: Comparison of marker orders between the three population-specific consolidated maps, generated by MapChart 2.1. Additional file 2: The BnaWAIT_01_2010a integrated map (by JoinMap) generated by MapChart 2.1.
\end{abstract}

Additional file 3: All the information of high-scoring segement pairs (HSP) of canonical markers against Arabidopsis gene models, Arabidopsis chromosomes and B. rapa sequenced BACs. The information of the BnaWAIT_10_2010a BnaWAIT map and markers aligned with Arabidopsis genes and chromosomes are also shown, so are the skeleton maps of each population.

Additional file 4: Dot-plots between the BnaWAIT map and all three population-specific maps for the remainder of all 19 LGs. The marker order of the vertical axis is from the BnaWAIT_01_2010a integrated map, and three marker orders of the horizontal axis are for the three population-specific maps.

Additional file 5: Integrated skeleton maps of representative markers generated by JoinMap 4.0 Round 1 and Round 2 under the algorithm of regression. The number of excluded representative markers from the map integration in these two rounds is reported. Spearman's rank correlation coefficients ( $r$ ) between the two integrated maps (map1 and map2) and the three population-specific maps (BnaSNDH, BnaSGDH and BnaDYDH) are also shown.

Additional file 6: Number of different sets of canonical marker assays using in the $B$. napus mapping, number of marker assays that show homology with Arabidopsis and number of homology regions in Arabidopsis with similarity to Brassica canonical marker assays.

Additional file 7: Summary of conserved collinearity blocks between the $B$. napus integrated map BnaWAIT_01_2010a and the Arabidopsis genome sequence.

Additional file 8: Description of mapping populations and genetic markers used in the map integration study, which are maintained in CropStoreDB. Corresponding references are also shown if available.

Additional file 9: Diagram of database interaction facilitating the map integration process and establishing links between genetic maps to DNA sequence information (e.g., TAIR9 genome or B. rapa $B A C s)$ via sequence-tagged marker sequences. CropStoreDB is used to manage data relating to Brassica genetics, including populations, genetic maps, genetic markers and their positions. SeqStoreDB is used to manage all publicly available Brassica sequences together with sequence data from private sources. AlignStoreDB is used to manage all the 
homology alignments between query Brassica sequences and target genomic or BAC sequences.

\section{Acknowledgements}

JW and GJK are funded by Biotechnology and Biological Sciences Research Council (BBRSC), and carried out this work in projects BBE0177971 and BBE0016101. We are grateful that Xiaowu Wang, IVF-CAAS, Beijing, who kindly provided us with pre-publication access to B. rapa Chiifu-401 genome scaffolds. We thank Ambrose Andongabo for the construction and maintenance of CropStoreDB online interface and SeqStoreDB.

\section{Author details}

1 Department of Plant Sciences, Rothamsted Research, Harpenden, AL5 2JQ, UK. ${ }^{2}$ Agriculture and Agri-Food Canada, 107 Science Place, Saskatoon, Saskatchewan, S7N 0X2, Canada. ${ }^{3}$ UMR 118 Amélioration des Plantes et Biotechnologies Végétales, INRA, BP 35327, 35653 Le Rheu Cedex, France. ${ }^{4}$ Centre for Haemato-Oncology, Barts Cancer Institute, Barts and The London School of Medicine and Dentistry, Charterhouse Square, London, EC1 M 6BQ, UK. ${ }^{5}$ Southern Cross Plant Sciences, Southern Cross University, Lismore, NSW 2480, Australia.

\section{Authors' contributions}

JW and GJK conceived the study. JW designed and performed the study, and wrote the paper. GJK designed and supervised the work, and wrote the paper. DJL, IAPP, CF and RD provided unpublished data and commented on the work. DJL contributed to editing the paper. PWCC curated the data. All authors read and approved the final manuscript.

\section{Received: 19 October 2010 Accepted: 9 February 2011}

Published: 9 February 2011

\section{References}

1. Yang YW, Lai KN, Tai PY, Li WH: Rates of nucleotide substitution in angiosperm mitochondrial DNA sequences and dates of divergence between Brassica and other angiosperm lineages. J Mol Evol 1999, 48(5):597-604

2. Koch MA, Haubold B, Mitchell-Olds T: Comparative evolutionary analysis of chalcone synthase and alcohol dehydrogenase loci in Arabidopsis, Arabis, and related genera (Brassicaceae). Mol Biol Evol 2000, 17(10):1483-1498.

3. Osborn TC, Kole C, Parkin IAP, Sharpe AG, Kuiper M, Lydiate DJ, Trick M: Comparison of flowering time genes in Brassica rapa, B-napus and Arabidopsis thaliana. Genetics 1997, 146(3):1123-1129.

4. Lan TH, DelMonte TA, Reischmann KP, Hyman J, Kowalski SP, McFerson J, Kresovich S, Paterson AH: An EST-enriched comparative map of Brassica oleracea and Arabidopsis thaliana. Genome Res 2000, 10(6):776-788.

5. Parkin IAP, Lydiate DJ, Trick M: Assessing the level of collinearity between Arabidopsis thaliana and Brassica napus for A-thaliana chromosome 5. Genome 2002, 45(2):356-366.

6. Lysak MA, Koch MA, Pecinka A, Schubert I: Chromosome triplication found across the tribe Brassiceae. Genome Res 2005, 15(4):516-525.

7. Parkin IAP, Gulden SM, Sharpe AG, Lukens L, Trick M, Osborn TC, Lydiate DJ: Segmental structure of the Brassica napus genome based on comparative analysis with Arabidopsis thaliana. Genetics 2005 171(2):765-781.

8. Lagercrantz U: Comparative mapping between Arabidopsis thaliana and Brassica nigra indicates that Brassica genomes have evolved through extensive genome replication accompanied by chromosome fusions and frequent rearrangements. Genetics 1998, 150(3):1217-1228.

9. Lukens L, Zou F, Lydiate D, Parkin I, Osborn T: Comparison of a Brassica oleracea genetic map with the genome of Arabidopsis thaliana. Genetics 2003, 164(1):359-372.

10. Panjabi $P$, Jagannath A, Bisht NC, Padmaja KL, Sharma S, Gupta V Pradhan AK, Pental D: Comparative mapping of Brassica juncea and Arabidopsis thaliana using Intron Polymorphism (IP) markers: homoeologous relationships, diversification and evolution of the $A, B$ and C Brassica genomes. Bmc Genomics 2008, 9.
11. Kim H, Choi SR, Bae J, Hong CP, Lee SY, Hossain MJ, Van Nguyen D, Jin M, Park BS, Bang JW, et al: Sequenced BAC anchored reference genetic map that reconciles the ten individual chromosomes of Brassica rapa. BmC Genomics 2009, 10.

12. Simillion C, Vandepoele $\mathrm{K}$, Van Montagu MCE, Zabeau M, Van de Peer $\mathrm{Y}$ : The hidden duplication past of Arabidopsis thaliana. P Natl Acad Sci USA 2002, 99(21):13627-13632.

13. Blanc $\mathrm{G}$, Hokamp K, Wolfe $\mathrm{KH}$ : A recent polyploidy superimposed on older large-scale duplications in the Arabidopsis genome. Genome Res 2003, 13(2):137-144.

14. Blanc $\mathrm{G}$, Wolfe $\mathrm{KH}$ : Functional divergence of duplicated genes formed by polyploidy during Arabidopsis evolution. Plant Cell 2004, 16(7):1679-1691.

15. Mun JH, Kwon SJ, Yang TJ, Seol YJ, Jin M, Kim JA, Lim MH, Kim JS, Baek S, Choi BS, et al: Genome-wide comparative analysis of the Brassica rapa gene space reveals genome shrinkage and differential loss of duplicated genes after whole genome triplication. Genome Biol 2009, 10(10).

16. Parkin IA, Sharpe AG, Keith DJ, Lydiate DJ: Identification of the $A$ and $C$ genomes of amphidiploid Brassica napus (oilseed rape). Genome 1995, 38(6):1122-1131.

17. Sharpe AG, Parkin IA, Keith DJ, Lydiate DJ: Frequent nonreciprocal translocations in the amphidiploid genome of oilseed rape (Brassica napus). Genome 1995, 38(6):1112-1121.

18. Uzunova M, Ecke W, Weissleder K, Robbelen G: Mapping the Genome of Rapeseed (Brassica-Napus L) .1. Construction of an Rflp Linkage Map and Localization of Qtls for Seed Glucosinolate Content. Theor App/ Genet 1995, 90(2):194-204.

19. Lombard V, Delourme R: A consensus linkage map for rapeseed (Brassica napus L.): construction and integration of three individual maps from DH populations. Theor Appl Genet 2001, 103(4):491-507.

20. Lowe AJ, Moule C, Trick M, Edwards KJ: Efficient large-scale development of microsatellites for marker and mapping applications in Brassica crop species. Theor Appl Genet 2004, 108(6):1103-1112.

21. Piquemal J, Cinquin E, Couton $F$, Rondeau C, Seignoret $E$, Doucet $I$, Perret D, Villeger MJ, Vincourt P, Blanchard P: Construction of an oilseed rape (Brassica napus L.) genetic map with SSR markers. Theor App/ Genet 2005, 111(8):1514-1523.

22. Delourme R, Falentin C, Huteau V, Clouet V, Horvais R, Gandon B, Specel S, Hanneton L, Dheu JE, Deschamps M, et al: Genetic control of oil content in oilseed rape (Brassica napus L.). Theor Appl Genet 2006, 113(7):1331-1345.

23. Qiu D, Morgan C, Shi J, Long Y, Liu J, Li R, Zhuang X, Wang Y, Tan X,

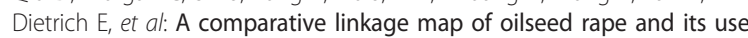
for QTL analysis of seed oil and erucic acid content. Theor App/ Genet 2006, 114(1):67-80.

24. Long Y, Shi J, Oiu D, Li R, Zhang C, Wang J, Hou J, Zhao J, Shi L, Park BS, et al: Flowering time quantitative trait loci analysis of oilseed Brassica in multiple environments and genomewide alignment with Arabidopsis. Genetics 2007, 177(4):2433-2444.

25. Delourme R, Piel N, Horvais R, Pouilly N, Domin C, Vallee P, Falentin C, Manzanares-Dauleux MJ, Renard M: Molecular and phenotypic characterization of near isogenic lines at QTL for quantitative resistance to Leptosphaeria maculans in oilseed rape (Brassica napus L.). Theor Appl Genet 2008, 117(7):1055-1067.

26. Suwabe $K$, Morgan C, Bancroft I: Integration of Brassica A genome genetic linkage map between Brassica napus and B. rapa. Genome 2008, 51(3):169-176

27. Cone KC, McMullen MD, Bi IV, Davis GL, Yim YS, Gardiner JM, Polacco ML, Sanchez-Villeda H, Fang Z, Schroeder SG, et al: Genetic, physical, and informatics resources for maize. On the road to an integrated map. Plant Physiol 2002, 130(4):1598-1605.

28. Falque $M$, Decousset $L$, Dervins $D$, Jacob AM, Joets J, Martinant JP, Raffoux X, Ribiere N, Ridel C, Samson D, et al: Linkage mapping of 1454 new maize candidate gene Loci. Genetics 2005, 170(4):1957-1966.

29. Song QJ, Marek LF, Shoemaker RC, Lark KG, Concibido VC, Delannay X, Specht JE, Cregan PB: A new integrated genetic linkage map of the soybean. Theor Appl Genet 2004, 109(1):122-128.

30. Choi IY, Hyten DL, Matukumalli LK, Song QJ, Chaky JM, Quigley CV, Chase K, Lark KG, Reiter RS, Yoon MS, et al: A soybean transcript map: Gene distribution, haplotype and single-nucleotide polymorphism analysis. Genetics 2007, 176(1):685-696. 
31. Wenzl P, Li HB, Carling J, Zhou MX, Raman H, Paul E, Hearnden P, Maier C, Xia L, Caig V, et al: A high-density consensus map of barley linking DArT markers to SSR, RFLP and STS loci and agricultural traits. Bmc Genomics 2006, 7.

32. Marcel TC, Varshney RK, Barbieri M, Jafary H, de Kock MJD, Graner A, Niks RE: A high-density consensus map of barley to compare the distribution of QTLs for partial resistance to Puccinia hordei and of defence gene homologues. Theor App/ Genet 2007, 114(3):487-500.

33. Varshney RK, Marcel TC, Ramsay L, Russell J, Roder MS, Stein N, Waugh R, Langridge P, Niks RE, Graner A: A high density barley microsatellite consensus map with 775 SSR loci. Theor Appl Genet 2007, 114(6):1091-1103.

34. Bhattramakki D, Dong JM, Chhabra AK, Hart GE: An integrated SSR and RFLP linkage map of Sorghum bicolor (L.) Moench. Genome 2000, 43(6):988-1002.

35. Haussmann BIG, Hess DE, Seetharama N, Welz HG, Geiger HH: Construction of a combined sorghum linkage map from two recombinant inbred populations using AFLP, SSR, RFLP, and RAPD markers, and comparison with other sorghum maps. Theor Appl Genet 2002, 105(4):629-637.

36. Mace ES, Rami JF, Bouchet $S$, Klein PE, Klein RR, Kilian A, Wenzl P, Xia L, Halloran K, Jordan DR: A consensus genetic map of sorghum that integrates multiple component maps and high-throughput Diversity Array Technology (DArT) markers. Bmc Plant Biol 2009, 9.

37. Singh K, Ghai M, Garg M, Chhuneja P, Kaur P, Schnurbusch T, Keller B, Dhaliwal HS: An integrated molecular linkage map of diploid wheat based on a Triticum boeoticum $\times$ T. monococcum RIL population. Theor Appl Genet 2007, 115(3):301-312.

38. Jing HC, Bayon C, Kanyuka K, Berry S, Wenzl P, Huttner E, Kilian A, Hammond-Kosack KE: DArT markers: diversity analyses, genomes comparison, mapping and integration with SSR markers in Triticum monococcum. Bmc Genomics 2009, 10:458.

39. Doligez A, Adam-Blondon AF, Cipriani G, Laucou V, Merdinoglu D, Meredith CP, Riaz S, Roux C, This P, Di Gaspero G: An integrated SSR map of grapevine based on five mapping populations. Theor Appl Genet 2006, 113(3):369-382.

40. Vezzulli S, Troggio M, Coppola G, Jermakow A, Cartwright D, Zharkikh A, Stefanini M, Grando MS, Viola R, Adam-Blondon AF, et al: A reference integrated map for cultivated grapevine (Vitis vinifera L.) from three crosses, based on 283 SSR and 501 SNP-based markers. Theor Appl Genet 2008, 117(4):499-511

41. Muchero W, Diop NN, Bhat PR, Fenton RD, Wanamaker S, Pottorff M, Hearne S, Cisse N, Fatokun C, Ehlers JD, et al: A consensus genetic map of cowpea [Vigna unguiculata (L) Walp.] and synteny based on EST-derived SNPs. P Natl Acad Sci USA 2009, 106(43):18159-18164.

42. Hong YB, Chen XP, Liang XQ, Liu HY, Zhou GY, Li SX, Wen SJ, Holbrook CC, Guo BZ: A SSR-based composite genetic linkage map for the cultivated peanut (Arachis hypogaea L.) genome. Bmc Plant Biol 2010, 10.

43. Hu J, Sadowski J, Osborn TC, Landry BS, Quiros CF: Linkage group alignment from four independent Brassica oleracea RFLP maps. Genome 1998, 41(2):226-235.

44. Beavis WD, Grant D: A Linkage Map Based on Information from 4 F2 Populations of Maize (Zea-Mays L). Theor App/ Genet 1991, 82(5):636-644.

45. Beavis WD, Grant D, Albertsen M, Fincher R: Quantitative Trait Loci for Plant Height in 4 Maize Populations and Their Associations with Qualitative Genetic-Loci. Theor App/ Genet 1991, 83(2):141-145.

46. Stam P: Construction of Integrated Genetic-Linkage Maps by Means of a New Computer Package - Joinmap. Plant J 1993, 3(5):739-744.

47. Jansen J, de Jong AG, van Ooijen JW: Constructing dense genetic linkage maps. Theor Appl Genet 2001, 102(6-7):1113-1122.

48. van Ooijen JW: JoinMap ${ }^{\oplus}$, Software for the calculation of genetic linkage maps in experimental populations. Wageningen, Netherlands: Kyazma B. V; 2006.

49. de Givry S, Bouchez M, Chabrier P, Milan D, Schiex T: CARHTA GENE: multipopulation integrated genetic and radiation hybrid mapping. Bioinformatics 2005, 21(8):1703-1704.

50. Wu Y, Close TJ, Lonardi S: On the accurate construction of consensus genetic maps. Comput Syst Bioinformatics Conf 2008, 7:285-296.

51. Yap IV, Schneider D, Kleinberg J, Matthews D, Cartinhour S, McCouch SR: A graph-theoretic approach to comparing and integrating genetic, physical and sequence-based maps. Genetics 2003, 165(4):2235-2247.
52. Jackson BN, Aluru S, Schnable PS: Consensus genetic maps: A graph theoretic approach. 2005 IEEE Computational Systems Bioinformatics Conference, Proceedings 2005, 35-43.

53. Arumuganathan K, Earle ED: Nuclear DNA content of some important plant species. Plant Molecular Biology Reporter 1991, 9(3):208-218.

54. Johnston JS, Pepper AE, Hall AE, Chen ZJ, Hodnett G, Drabek J, Lopez R, Price HJ: Evolution of genome size in Brassicaceae. Ann Bot-London 2005, 95(1):229-235.

55. Fourmann M, Barret P, Froger N, Baron C, Charlot F, Delourme R, Brunel D: From Arabidopsis thaliana to Brassica napus: development of amplified consensus genetic markers (ACGM) for construction of a gene map. Theor Appl Genet 2002, 105(8):1196-1206.

56. Wang J, Long Y, Wu BD, Liu J, Jiang CC, Shi L, Zhao JW, King GJ, Meng JL: The evolution of Brassica napus FLOWERING LOCUST paralogues in the context of inverted chromosomal duplication blocks. Bmc Evol Biol 2009, 9.

57. Ryder CD, Smith LB, Teakle GR, King GJ: Contrasting genome organisation: two regions of the Brassica oleracea genome compared with collinear regions of the Arabidopsis thaliana genome. Genome 2001, 44(5):808-817.

58. Howell EC, Armstrong SJ, Barker GC, Jones GH, King GJ, Ryder CD, Kearsey MJ: Physical organization of the major duplication on Brassica oleracea chromosome $\mathrm{O6}$ revealed through fluorescence in situ hybridization with Arabidopsis and Brassica BAC probes. Genome 2005, 48(6):1093-1103

59. Consortium BrGSP: The genome of the mesohexaploid crop species Brassica rapa. In preparation 2010.

60. Close TJ, Bhat PR, Lonardi S, Wu Y, Rostoks N, Ramsay L, Druka A, Stein N, Svensson JT, Wanamaker $S$, et al: Development and implementation of high-throughput SNP genotyping in barley. Bmc Genomics 2009, 10:582.

61. Yu J, Kohel RJ, Smith CW: The construction of a tetraploid cotton genome wide comprehensive reference map. Genomics 2010, 95(4):230-240

62. Lukaszewski AJ, Curtis CA: Physical Distribution of Recombination in BGenome Chromosomes of Tetraploid Wheat. Theor App/ Genet 1993, 86(1):121-127.

63. Stephan W, Langley $\mathrm{CH}$ : DNA polymorphism in Lycopersicon and crossing-over per physical length. Genetics 1998, 150(4):1585-1593.

64. Akhunov ED, Goodyear AW, Geng S, Qi LL, Echalier B, Gill BS, Gustafson JP, Lazo G, Chao SM, et al: The organization and rate of evolution of wheat genomes are correlated with recombination rates along chromosome arms. Genome Res 2003, 13(5):753-763.

65. Wu JZ, Mizuno H, Hayashi-Tsugane M, Ito Y, Chiden Y, Fujisawa M, Katagiri S, Saji S, Yoshiki S, Karasawa W, et al: Physical maps and recombination frequency of six rice chromosomes. Plant $J$ 2003, 36(5):720-730

66. Anderson LK, Salameh N, Bass HW, Harper LC, Cande WZ, Weber G, Stack SM: Integrating genetic linkage maps with pachytene chromosome structure in maize. Genetics 2004, 166(4):1923-1933.

67. Fu H, Zheng Z, Dooner HK: Recombination rates between adjacent genic and retrotransposon regions in maize vary by 2 orders of magnitude. Proc Natl Acad Sci USA 2002, 99(2):1082-1087.

68. SanMiguel PJ, Ramakrishna W, Bennetzen JL, Busso CS, Dubcovsky J: Transposable elements, genes and recombination in a $215-\mathrm{kb}$ contig from wheat chromosome 5A(m). Funct Integr Genomics 2002, 2(1-2):70-80.

69. Wei F, Wing RA, Wise RP: Genome dynamics and evolution of the Mla (powdery mildew) resistance locus in barley. Plant Cell 2002, 14(8):1903-1917.

70. Wright SI, Agrawal N, Bureau TE: Effects of recombination rate and gene density on transposable element distributions in Arabidopsis thaliana. Genome Res 2003, 13(8):1897-1903.

71. Jensen-Seaman MI, Furey TS, Payseur BA, Lu YT, Roskin KM, Chen CF, Thomas MA, Haussler D, Jacob HJ: Comparative recombination rates in the rat, mouse, and human genomes. Genome Res 2004, 14(4):528-538.

72. Marais G, Charlesworth B, Wright SI: Recombination and base composition: the case of the highly self-fertilizing plant Arabidopsis thaliana. Genome Biol 2004, 5(7).

73. Mezard C: Meiotic recombination hotspots in plants. Biochem Soc T 2006, 34:531-534.

74. Drouaud J, Camilleri C, Bourguignon PY, Canaguier A, Berard A, Vezon D, Giancola S, Brunel D, Colot V, Prum B, et al: Variation in crossing-over 
rates across chromosome 4 of Arabidopsis thaliana reveals the presence of meiotic recombination "hot spots". Genome Res 2006, 16(1):106-114.

75. Lander ES, Green P, Abrahamson J, Barlow A, Daly MJ, Lincoln SE, Newberg LA: MAPMAKER: an interactive computer package for constructing primary genetic linkage maps of experimental and natural populations. Genomics 1987, 1(2):174-181.

76. Lincoln SE, Lander ES: Systematic detection of errors in genetic linkage data. Genomics 1992, 14(3):604-610.

77. Howad W, Yamamoto T, Dirlewanger E, Testolin R, Cosson P, Cipriani G, Monforte AJ, Georgi L, Abbott AG, Arus P: Mapping with a few plants: using selective mapping for microsatellite saturation of the Prunus reference map. Genetics 2005, 171(3):1305-1309.

78. Choi SR, Teakle GR, Plaha P, Kim JH, Allender CJ, Beynon E, Piao ZY, Soengas $P$, Han TH, King GJ, et al: The reference genetic linkage map for the multinational Brassica rapa genome sequencing project. Theor Appl Genet 2007, 115(6):777-792.

79. Kim JS, Chung TY, King GJ, Jin M, Yang TJ, Jin YM, Kim HI, Park BS: A sequence-tagged linkage map of Brassica rapa. Genetics 2006, 174(1):29-39.

80. Farber $C R$, Medrano JF: Putative in silico mapping of DNA sequences to livestock genome maps using SSLP flanking sequences. Anim Genet 2003, 34(1):11-18.

81. Farber CR, Medrano JF: Identification of putative homology between horse microsatellite flanking sequences and cross-species ESTs, mRNAs and genomic sequences. Anim Genet 2004, 35(1):28-33.

82. Cho K, O'Neill CM, Kwon SJ, Yang TJ, Smooker AM, Fraser F, Bancroft !: Sequence-level comparative analysis of the Brassica napus genome around two stearoyl-ACP desaturase loci. Plant J 2010, 61(4):591-599.

83. Schranz ME, Lysak MA, Mitchell-Olds T: The ABC's of comparative genomics in the Brassicaceae: building blocks of crucifer genomes. Trends Plant Sci 2006, 11(11):535-542.

doi:10.1186/1471-2164-12-101

Cite this article as: Wang et al.: Integration of linkage maps for the Amphidiploid Brassica napus and comparative mapping with Arabidopsis and Brassica rapa. BMC Genomics 2011 12:101.

\section{Submit your next manuscript to BioMed Central and take full advantage of:}

- Convenient online submission

- Thorough peer review

- No space constraints or color figure charges

- Immediate publication on acceptance

- Inclusion in PubMed, CAS, Scopus and Google Scholar

- Research which is freely available for redistribution

Submit your manuscript at www.biomedcentral.com/submit 